International Journal of Modern Physics A

(C) World Scientific Publishing Company

\title{
AN UPDATED ANALYSIS ON THE RISE OF THE HADRONIC TOTAL CROSS-SECTION AT THE LHC ENERGY REGION
}

\author{
M. J. MENON and P. V. R. G. SILVA \\ Universidade Estadual de Campinas - UNICAMP \\ Instituto de Física Gleb Wataghin \\ 13083-859 Campinas, SP, Brazil \\ (menon@if.unicamp.br, precchia@ifi.unicamp.br)
}

\begin{abstract}
A forward amplitude analysis on $p p$ and $\bar{p} p$ elastic scattering above $5 \mathrm{GeV}$ is presented. The dataset includes the recent high-precision TOTEM measurements of the $p p$ total and elastic (integrated) cross-sections at $7 \mathrm{TeV}$ and $8 \mathrm{TeV}$. Following previous works, the leading high-energy contribution for the total cross-section $\left(\sigma_{t o t}\right)$ is parametrized as $\ln ^{\gamma}\left(s / s_{h}\right)$, where $\gamma$ and $s_{h}$ are free real fit parameters. Singly-subtracted derivative dispersion relations are used to connect $\sigma_{t o t}$ and the rho parameter $(\rho)$ in an analytical way. Different fit procedures are considered, including individual fits to $\sigma_{\text {tot }}$ data, global fits to $\sigma_{t o t}$ and $\rho$ data, constrained and unconstrained data reductions. The results favor a rise of the $\sigma_{\text {tot }}$ faster than the log-squared bound by Froissart and Martin at the LHC energy region. The parametrization for $\sigma_{t o t}$ is extended to fit the elastic cross-section $\left(\sigma_{e l}\right)$ data with satisfactory results. The analysis indicates an asymptotic ratio $\sigma_{e l} / \sigma_{t o t}$ consistent with $1 / 3$ (as already obtained in a previous work). A critical discussion on the correlation, practical role and physical implications of the parameters $\gamma$ and $s_{h}$ is presented. The discussion confronts the 2002 prediction of $\sigma_{t o t}$ by the COMPETE Collaboration and the recent result by the Particle Data Group (2012 edition of the Review of Particle Physics). Some conjectures on possible implications of a fast rise of the proton-proton total cross-section at the highest energies are also presented.
\end{abstract}

Keywords: Hadron-induced high- and super-high-energy interactions; Total crosssections; Asymptotic problems and properties

PACS numbers: 13.85.-t, 13.85.Lg, 11.10.Jj

\section{Introduction}

The theoretical and phenomenological descriptions of the energy dependence of the hadronic total cross-section at high energies have been a fundamental and longstanding problem. Given that the optical theorem connects the total cross-section with the imaginary part of the forward elastic scattering amplitude, the main theoretical difficulty concerns the lack of a pure (model-independent) nonperturbative QCD description of the soft scattering states, in particular the elastic channel in the forward direction. In this section, we shortly recall some results on the rise of the total cross-section of interest in this paper, followed by the plan of the paper.

Historical formal analyses by Froissart, Lukaszuk and Martir ${ }^{1+4}$ have established 
the famous asymptotic bound for the rise of the total cross-section,

$$
\sigma_{t o t}(s) \leq c \ln ^{2} \frac{s}{s_{0}}
$$

where $s$ is the center-of-mass energy squared, $c \leq \pi / m_{\pi}^{2} \approx 60 \mathrm{mb}$ and $s_{0}$ is a constant. After the Martin derivation in the context of the axiomatic quantum field theory ${ }^{2}$ the $\log$-squared bound has played a central role in model constructions, aimed to treat, interpret and describe strong interactions. Recently, Azimov has argued that it is not obvious that QCD can be considered an axiomatic theory and has also established new constraints for the rate of increase of $\sigma_{t o t}(s) ! \underline{5}$ The formalism allows to conclude that, depending on the behavior of the amplitude in the nonphysical region, the total hadronic cross-section may rise faster than the $\log$-squared bound, without violation of unitarity ${ }^{5}$ (see also Refs. [6] and 7 ).

The dependence of the total cross-section on the energy has been usually investigated through forward amplitude analysis. This approach is characterized by different analytical parametrizations for $\sigma_{t o t}(s)$ and global data reductions including the $\rho$ parameter (ratio between the real and imaginary parts of the forward amplitude) by means of singly-subtracted dispersion relations. In 2002, the COMPETE Collaboration developed a detailed analysis on different functional forms, using a ranking procedure and including several reactions and different energy cutoffs $\frac{8}{8}[9$ The analysis favored the asymptotic form $\ln ^{2}\left(s / s_{0}\right)$, when compared with $\ln \left(s / s_{0}\right)$ or powers $\left[s / s_{0}\right]^{\epsilon}$ with $\epsilon>0$, a conclusion corroborated by subsequent works 10 - 13 After that, data reductions with the COMPETE highest-rank parametrization, in agreement with the log-squared bound and all the experimental data available, became standard reference in the Review of Particle Physics by the Particle Data Group (PDG) up to the 2010 edition.14

In the experimental context, the recent results from the LHC, reaching the highest energy region, opened new expectations in the phenomenological and theoretical contexts. However, the first results on $p p$ elastic, diffractive and nondiffractive processes by the TOTEM Collaboration have indicated noticeable discrepancies with standard predictions from representative phenomenological models $\frac{15}{17}$ In general grounds, this novel experimental information seems yet unable to select or exclude classes of phenomenological approaches and/or theoretical pictures in a clear and conclusive way.

In what concerns the $p p$ total cross-section, the first $7 \mathrm{TeV}$ TOTEM highprecision measurement has been obtained with small bunches and a luminositydependent method, indicating

$$
\sigma_{\text {tot }, 1}(7 \mathrm{TeV})=98.3 \pm 2.8 \mathrm{mb} \text { (small bunches / luminosity-dependent) } \underline{16}
$$

In particular and for our purposes, the COMPETE prediction (published 11 years ago) is in good agreement with this datum $\left[\frac{16}{16}\right.$ However, contrasting with this striking result, the 2012 edition of the Review of Particle Physics by the PDG has shown that with an updated dataset, including the above TOTEM datum, the fit with the highest-rank parametrization selected by the COMPETE Collaboration 
disagrees with the TOTEM result (Figure 46.10 in Ref. 18). Both results (COMPETE 2002 and PDG 2012) are displayed in Figure 1 and will be discussed along the paper.

In 2011 and 2012, Fagundes, Menon and Silva 20 have revisited a parametrization for the total cross-section introduced by Amaldi et al. in the seventies, ${ }^{21}$ characterized by using the exponent in the high-energy leading logarithm contribution as a free real fit parameter. Based on different fit procedures, variants and data ensembles from $p p$ and $\bar{p} p$ forward scattering at $\sqrt{s} \geq 5 \mathrm{GeV}$, the authors have shown that with the inclusion of the $7 \mathrm{TeV}$ TOTEM result in the dataset, several statistically consistent solutions are obtained with the exponent greater than 2, suggesting, therefore, a faster-than-squared-logarithm rise for the total cross-section.19]20]

More recently, the TOTEM Collaboration has obtained four new high-precision measurements for the total cross-section, with three data at $7 \mathrm{TeV}$ and one datum at $8 \mathrm{TeV} \cdot 22 \sqrt{24}$ The results and experimental conditions can be summarized as follows:

$$
\begin{array}{ll}
\sigma_{t o t, 2}(7 \mathrm{TeV})=98.6 \pm 2.2 \mathrm{mb} & \text { (large bunches } / \text { luminosity-dependent) }{ }^{22} \\
\sigma_{t o t, 3}(7 \mathrm{TeV})=99.1 \pm 4.3 \mathrm{mb} & (\rho \text { independent }),{ }^{23} \\
\sigma_{t o t, 4}(7 \mathrm{TeV})=98.0 \pm 2.5 \mathrm{mb} & \text { (luminosity-independent) }{ }^{23} \\
\sigma_{t o t}(8 \mathrm{TeV})=101.7 \pm 2.9 \mathrm{mb} & \text { (luminosity-independent) }{ }^{24}
\end{array}
$$

In this paper, we revisit, once more, the Amaldi et al. parametrization, now taking into account these recent measurements of the $p p$ total cross-section at $7 \mathrm{TeV}$ and $8 \mathrm{TeV}$. The general strategy follows the approach developed in our previous analysis $\frac{19}{20}$ In addition to using the new data, we will also explore the practical role and the physical meaning of the free fit parameters associated with the highenergy leading contribution. In particular, we discuss the differences between the 2002 COMPETE prediction and the 2012 PDG result in the phenomenological context (Reggeon/Pomeron exchanges). As in Refs. 19 and 20, the analysis is also restricted to $p p$ and $\bar{p} p$ data at $\sqrt{s} \geq 5 \mathrm{GeV}$, the same energy cutoff used by the COMPETE Collaboration ${ }^{8 / 9}$ and in the last PDG versions. ${ }^{14}[18$ Our analysis and results favor, once more, a rise of the hadronic total cross-section faster than the logsquared bound at the LHC energy region. An extension of the parametrization to fit the elastic cross-section data allows us to infer the asymptotic ratio between the elastic and total cross-sections. The result is statistically consistent with a rational limit of $1 / 3$ (as previously obtained in Ref. 20). Some comments and conjectures on the possible implications of a faster-than-squared-logarithm rise for the total cross-section are also presented.

The paper is organized as follows. In section 2, we display the analytical parametrization for $\sigma_{\text {tot }}(s)$ and the formula connecting this quantity with $\rho(s)$, using singly-subtracted derivative dispersion relations, $\frac{19}{19}$ the fit procedures and strategies are also outlined. In section 3 we present the fit results with datasets up to $\sqrt{s}_{\text {max }}$ $=7 \mathrm{TeV}$ and $\sqrt{s}_{\text {max }}=8 \mathrm{TeV}$, in the cases of unconstrained and constrained data 
reductions, individual fits to $\sigma_{\text {tot }}$ data and global fits to $\sigma_{t o t}$ and $\rho$ data. A discussion on all the obtained results, with focus on the high-energy leading contribution parameters, is presented in section 4. The extension to the elastic cross-section data is treated in section 5 and discussions on the possibility of a remarkable fast rise of the total cross-section at the LHC energy region are presented in section 6. The conclusions and some final remarks are the contents of section 7 .

\section{Analytical Parametrization and Fit Procedures}

In this section, after introducing the formulas to be employed in our data reductions, namely the parametrization for $\sigma_{\text {tot }}(s)$ and the analytical expression for $\rho(s)$ from derivative dispersion relations, we outline some important points on the fit procedures and strategies.

\subsection{Analytical parametrization and dispersion relation result}

We consider the analytical parametrization for the $p p$ and $\bar{p} p$ total cross-section introduced by Amaldi et al. in 1970s $\mathrm{s}^{21}$ and also employed by the UA4/2 Collaboration in 1990s. ${ }^{25}$ It consists of two components, associated with low-energy $(L E)$ and high-energy $(H E)$ contributions:

$$
\sigma_{t o t}(s)=\sigma_{L E}(s)+\sigma_{H E}(s) .
$$

The first term accounts for the decrease of the total cross-section and the differences between $p p$ and $\bar{p} p$ scattering at low energies. In this paper it is expressed by

$$
\sigma_{L E}(s)=a_{1}\left[\frac{s}{s_{l}}\right]^{-b_{1}}+\tau a_{2}\left[\frac{s}{s_{l}}\right]^{-b_{2}},
$$

where $\tau=-1(+1)$ for $p p(\bar{p} p)$ scattering, $s_{l}=1 \mathrm{GeV}^{2}$ is fixed, and $a_{1}, b_{1}, a_{2}$ and $b_{2}$ are free fit parameters.

The second term accounts for the rising of the cross-section at higher energies and is given by

$$
\sigma_{H E}(s)=\alpha+\beta \ln ^{\gamma} \frac{s}{s_{h}},
$$

where $\alpha, \beta, \gamma$ and $s_{h}$ are free real parameters.

For further reference, we briefly recall that, in the context of the Regge-Gribov theory, the decreasing $\sigma_{L E}(s)$ contribution is associated with Reggeon exchanges: $b_{1}$ and $b_{2}$ correspond to the intercept of the trajectories and $a_{1}, a_{2}$ to the Reggeon strengths (residues). The $\sigma_{H E}(s)$ term simulates the rise of the total cross-section and is associated with the Pomeron exchange. For example, for $\gamma=1$, the constant plus $\ln s$ terms correspond to a double pole at $J=1$ and for $\gamma=2$ a triple pole (expressed by $\ln ^{2} s, \ln s$ and the constant terms) ${ }^{8}$ For our purposes it is important to note that for $\gamma=2$, parametrization (Eqs. (1)-(3)) has the same analytical structure of the highest-rank parametrization selected by the COMPETE Collaboration $\frac{8}{[9}$ 
The analytical connection with the $\rho$ parameter is obtained using singlysubtracted derivative dispersion relations in the operator expansion form introduced by Kang and Nicolescu ${ }^{26}$ (also discussed in Ref. 27). In terms of the parametrization (1)-(3) for $p p$ and $\bar{p} p$ scattering, the analytical results for $\rho(s)$ read $[19[20$

$$
\begin{aligned}
\rho(s) & =\frac{1}{\sigma_{\text {tot }}(s)}\left\{\frac{K}{s}-a_{1} \tan \left(\frac{\pi b_{1}}{2}\right)\left[\frac{s}{s_{l}}\right]^{-b_{1}}+\mathcal{A} \ln ^{\gamma-1}\left(\frac{s}{s_{h}}\right)\right. \\
& \left.+\mathcal{B} \ln ^{\gamma-3}\left(\frac{s}{s_{h}}\right)+\mathcal{C} \ln ^{\gamma-5}\left(\frac{s}{s_{h}}\right)+\tau a_{2} \cot \left(\frac{\pi b_{2}}{2}\right)\left[\frac{s}{s_{l}}\right]^{-b_{2}}\right\},
\end{aligned}
$$

where $K$ is the subtraction constant,

$$
\begin{aligned}
& \mathcal{A}=\frac{\pi}{2} \beta \gamma, \quad \mathcal{B}=\frac{1}{3}\left[\frac{\pi}{2}\right]^{3} \beta \gamma[\gamma-1][\gamma-2], \\
& \mathcal{C}=\frac{2}{15}\left[\frac{\pi}{2}\right]^{5} \beta \gamma[\gamma-1][\gamma-2][\gamma-3][\gamma-4],
\end{aligned}
$$

and, as before, $\tau=-1(+1)$ for $p p(\bar{p} p)$ scattering.

\subsection{Fit procedures and strategies}

The fit procedures and methodology, as well as the role and applicability of the subtraction constant in the derivative dispersion relation approach, have been discussed in detail in Refs. 19 and 20. Here we recall and also outline some important points of interest in this work, especially the introduction of a representation for the parameter $s_{h}$.

\subsubsection{Dataset}

Our goal is to investigate the rise of $\sigma_{\text {tot }}$ at the highest energy region and its asymptotic behavior. For that reason, we limit the analysis to particle-particle and antiparticle-particle collisions corresponding only to the largest energy interval with available data, namely $p p$ and $\bar{p} p$ scattering. Although somewhat restrictive, the main point is that this choice allows the investigation of possible high-energy effects that may be unrelated to the trends of the lower energy data on other reactions (as the constraints dictated by a supposed universal behavior).

The input dataset for fits concerns only accelerator data on $\sigma_{\text {tot }}$ and $\rho$ from $p p$ and $\bar{p} p$ scattering, covering the energy region from $5 \mathrm{GeV}$ up to $8 \mathrm{TeV}$. The energy cutoff is the same employed in the COMPETE and PDG analyses. ${ }^{18}$ The data below $7 \mathrm{TeV}$ have been collected from the PDG database, $\frac{18}{18}$ without any kind of data selection or sieve procedure. Statistical and systematic errors have been added in quadrature. Estimations of the $p p$ total cross-section from cosmic-ray experiments will be displayed in the figures as illustrative results. The TOTEM estimation for $\rho$ at $7 \mathrm{TeV}^{23}$ is also displayed as illustration. All the references on these data and estimations can be found in Ref. 20 . 


\subsubsection{Nonlinearity and feedback values}

Because the nonlinearity of the fit demands a choice for the initial values (feedbacks) for all free parameters $\stackrel{28}{28}$ different choices have been tested and discussed in our previous analyses $\frac{19}{20}$ (see also Ref. 29). Here, to initialize our parametric set, we consider only the values of the fit results in the updated 2012 PDG version, obtained with the highest-rank COMPETE parametrization $\frac{18}{18}$

This choice is based on the arguments that follow. The PDG data reductions have been developed with $\gamma$ fixed to 2. Due to the strong correlation among all the fit parameters (to be discussed in Subsec. 4.1), their final fit values are, therefore, consequences of this condition $(\gamma=2)$. In this sense, initializing the parametrization with these values can be considered a "conservative" choice. Moreover, since in the present analysis $\gamma$ is treated as a free parameter in fits including the recent TOTEM measurements, this choice allows us to investigate possible departures from the standard/canonical assumption $\gamma=2$.

However, it should be noted that the PDG and COMPETE analyses include different collision processes (meson- $p$, baryon- $p$, among others) and also tests on universality. Therefore our dataset, restricted to $p p$ and $\bar{p} p$ scattering, corresponds to only a subset of the ensemble employed in the global analysis by the COMPETE and PDG. Despite this, we understand that with this choice for the feedbacks, we initialize our parametrization with a statistically and physically meaningful input, contributing to the search for a consistent fit solution (see also Subsec. 2.2.4 below).

The values of the feedback parameters to be used in our fits are shown in the third column of Table 1. For further reference, we display in Table 1 the values of the parameters obtained with the highest-rank parametrization (Eqs. (1)-(3) with $\gamma$ $=2$ ) in both the 2002 COMPETE analysis (Table VIII in Ref. 8) and the 2012 PDG version (Table 46.2 in Ref. 18), which is based on an updated dataset including the first $7 \mathrm{TeV}$ TOTEM datum. In the last case, the values of the parameters $a_{1}$ and $a_{2}$ correspond to our normalization of Eq. (2), namely $s_{l}=1 \mathrm{GeV}^{2}$ fixed (which is different from the normalization adopted in Ref. 18). The corresponding results for $\sigma_{t o t}(s)$ and uncertainty regions (evaluated through propagation from the errors in Table 1) are shown in Figure 1, together with the experimental information (in this figure only the first $7 \mathrm{TeV}$ TOTEM measurement is displayed, as in Ref. 18). We shall return to these results along the paper.

\subsubsection{Individual and global fits}

Global fits to $\sigma_{\text {tot }}$ and $\rho$ data demand the use of dispersion relations with one subtraction and therefore the introduction of one more free parameter, the subtraction constant. As already discussed in Refs. 19 and 20 this parameter does not have a physical interpretation as opposed to the parameters present in the total cross-section parametrization, which are associated with Reggeon and Pomeron exchanges. Moreover, several authors have criticized the usual methods to extract $\rho$ (see Subsec. 2.2 in Ref. 19) and, in addition, due to the correlation among all the 
Table 1. Fit results through parametrization (1-3) with $\gamma=2$ obtained in the COMPETE and PDG analyses 918 The parameters $a_{1}, a_{2}, \alpha$ and $\beta$ are in $\mathrm{mb}, s_{h}$ in $\mathrm{GeV}^{2}, b_{1}, b_{2}$ are dimensionless $\left(s_{l}\right.$ $=1 \mathrm{GeV}^{2}$ ).

\begin{tabular}{ccc}
\hline & COMPETE 2002 (Ref. 9) & PDG 2012 (Ref. [8) \\
\hline$a_{1}$ & $42.1 \pm 1.3$ & $46.07 \pm 0.76$ \\
$b_{1}$ & $0.467 \pm 0.015$ & $0.462 \pm 0.002$ \\
$a_{2}$ & $32.19 \pm 0.94$ & $34.02 \pm 0.63$ \\
$b_{2}$ & $0.5398 \pm 0.0064$ & $0.550 \pm 0.005$ \\
$\alpha$ & $35.83 \pm 0.40$ & $34.71 \pm 0.15$ \\
$\beta$ & $0.3152 \pm 0.0095$ & $0.265 \pm 0.050$ \\
$s_{h}$ & $34.0 \pm 0.54$ & $16.21 \pm 0.16$ \\
\hline
\end{tabular}

fit parameters, the $\rho$ inclusion in global fit constraints the rise of the total crosssection, as demonstrated in Refs. 20, 30, 31 and references therein. Despite these disadvantages, we shall here consider both individual fits to $\sigma_{\text {tot }}$ data through Eqs. (1)-(3) and global fits to $\sigma_{\text {tot }}$ and $\rho$ data using Eqs. (1)-(5).

\subsubsection{Minimization and statistics}

The data reductions have been performed with the objects of the class TMinuit of the ROOT Framework ${ }^{32}$ We have employed the default MINUIT error analysis ${ }^{33}$ with the selective criteria that follow. In the minimization program a Confidence Level of one standard deviation was adopted in all fits $(\mathrm{UP}=1)$. In each test of fit, successive running of the MIGRAD have been considered (up to 5,000 calls), until full convergence has been reached, with the smallest FCN (chi-squared) and requiring Estimated Distance to Minimum $(\mathrm{EDM})<10^{-4}$, adequate for the one sigma CL. In some cases the MINOS algorithm and strategies 1 and 2 have also been employed to check the MIGRAD result. In addition, the error in the parameters should not exceed the central value. The error matrix provides the variances and covariances associated with each free parameter, which are used in the analytic evaluation of the uncertainty regions in the fitted and predicted quantities, by means of standard error propagation procedures ${ }^{28}$

To quantify goodness of fit we will resort to chi-square per degree of freedom $\left(\chi^{2} / \mathrm{DOF}\right)$ and the corresponding integrated probability, $P\left(\chi^{2}\right) \stackrel{28}{[28}$ The goal is not to compare or select fit procedures or fit results but only to check the statistical consistence of the data reductions in a reasonable way.

\subsubsection{Unconstrained and constrained fits}

The consideration that the exponent $\gamma$ in the leading logarithm component is a real (not integer) free fit parameter leads to some special consequences and particular conditions. These aspects, including the effects of the correlation between the free parameters $\gamma$ and $s_{h}$ in data reductions, will be discussed in some detail in Sec. 4 . after the presentation of our fit results. In order to implement and facilitate that 
discussion, we introduce here a useful representation for the high-energy scaling factor $s_{h}$, which will lead us to distinguish between unconstrained and constrained fits, as explained in what follows.

The representation is based on two arguments: (i) the reference to $s_{h}$ only as a (unknown) constant in the Froissart-Martin derivation of the bound; (ii) the reasonable physical conjecture that this factor might be proportional to the energy threshold for the scattering states (above the resonance region), namely $s_{h} \propto\left(m_{p}+m_{p}\right)^{2}$, where $m_{p}$ is the proton mass. In this case, we can represent the scaling factor by

$$
s_{h}=\delta\left[4 m_{p}^{2}\right], \quad 4 m_{p}^{2}=3.521 \mathrm{GeV}^{2},
$$

where $\delta$ is a real dimensionless parameter and $\delta=1$ at the physical threshold.

With this representation, we can distinguish two physical conditions in data reductions: either to consider $\delta$ indeed as a free fit parameter (equivalently, $s_{h}$ a free fit parameter) or to assume $\delta=1$ (equivalently, to fix $s_{h}=4 m_{p}^{2}$, the energy threshold). In what follows we shall denote these two variants by unconstrained fits ( $\delta$ free) and constrained fits ( $\delta=1$ fixed).

Here, as in the COMPETE and PDG analyses, we shall treat $s_{h}$ as a constant (with the above representation). However, it should be noted that the possibility of a slow rise of $s_{h}$ with $s$ (for large $s$ ) has been discussed by some authors, as for example in Refs. 5 and 34 .

\subsubsection{Ensembles and feedbacks}

As mentioned earlier, our dataset consists of $p p$ and $\bar{p} p$ accelerator data at $\sqrt{s} \geq$ $5 \mathrm{GeV}$. In order to investigate the effect in the fits associated with the TOTEM measurement at $8 \mathrm{TeV}$, as compared with those at $7 \mathrm{TeV}$, we shall consider two data ensembles. The first one with data up to $7 \mathrm{TeV}$ (including the four measurements) and a second one adding the $8 \mathrm{TeV}$ datum. For reference, we will denote these two variants by $\sqrt{s}_{\text {max }}=7 \mathrm{TeV}$ ensemble and $\sqrt{s}_{\max }=8 \mathrm{TeV}$ ensemble, respectively.

For the $\sqrt{s}_{\max }=7 \mathrm{TeV}$ ensemble, we use as feedback the values of the parameters from the 2012 PDG version, displayed in the third column of Table 1 In the case of global fits to $\sigma_{\text {tot }}$ and $\rho$ data, we consider $K=0$ for the initial value of the subtraction constant ${ }^{18}$ The fit results with this ensemble are then used as feedback to initialize the parametrization with the $\sqrt{s}_{\max }=8 \mathrm{TeV}$ ensemble, in each one of the variants considered (individual, global, unconstrained and constrained cases).

\section{Fit Results}

Summarizing the variants discussed in the last section, we select two ensembles of accelerator data on $\sigma_{\text {tot }}$ and $\rho$ at $\sqrt{s} \geq 5 \mathrm{GeV}$, one up to $\sqrt{s}_{\text {max }}=7 \mathrm{TeV}$ and another one up to $\sqrt{s}_{\max }=8 \mathrm{TeV}$. In each case we consider both unconstrained fits $\left(s_{h}\right.$ or $\delta$ in Eq.(6) as free fit parameter) and constrained fits $\left(s_{h}=4 m_{p}^{2}\right.$ or $\delta=1$ fixed), treating also both individual fits to $\sigma_{\text {tot }}$ data through Eqs. (1)-(3) and global fits to $\sigma_{t o t}$ and $\rho$ data, using Eqs. (1)-(5). In what follows we present the fit results 
and discuss only the accordance with the selective criteria outlined in Subsec. 2.2.4 The physical aspects and implications involved are addressed in Sec. 4.

\section{1. $\sqrt{s}_{\text {max }}=7 \mathrm{TeV}$ ensemble}

For this ensemble all data reductions presented satisfactory agreement with the selective criteria. The fit results and statistical information are displayed in Table 2. The curves, uncertainty regions (from error propagation) and experimental information in the case of global fits to $\sigma_{\text {tot }}$ and $\rho$ data are shown in Fig. 2 for the unconstrained fit and in Fig. 3 for the constrained one.

Table 2. $\sqrt{s}_{\max }=7 \mathrm{TeV}$ Ensemble. Fit results using Equations (1)-(3) for the $\sigma_{t o t}$ data and Equations (1)-(5) for the $\sigma_{\text {tot }}$ and $\rho$ data in the case of unconstrained $\left(s_{h}\right.$ free $)$ and constrained $\left(s_{h}=4 m_{p}^{2}\right)$ data reductions. Units as in Table 1

\begin{tabular}{ccccc}
\hline & \multicolumn{2}{c}{ Unconstrained $(\delta$ free $)$} & \multicolumn{2}{c}{ Constrained $(\delta=1)$} \\
& $\sigma_{\text {tot }}$ & $\sigma_{\text {tot }}$ and $\rho$ & $\sigma_{\text {tot }}$ & $\sigma_{\text {tot }}$ and $\rho$ \\
\hline$a_{1}$ & $59.5 \pm 8.8$ & $56.7 \pm 6.4$ & $60.5 \pm 7.8$ & $59.1 \pm 4.9$ \\
$b_{1}$ & $0.553 \pm 0.063$ & $0.528 \pm 0.062$ & $0.526 \pm 0.074$ & $0.500 \pm 0.054$ \\
$a_{2}$ & $33.2 \pm 2.2$ & $34.0 \pm 2.0$ & $33.2 \pm 2.3$ & $34.1 \pm 1.9$ \\
$b_{2}$ & $0.541 \pm 0.015$ & $0.547 \pm 0.013$ & $0.541 \pm 0.016$ & $0.547 \pm 0.013$ \\
$\alpha$ & $35.53 \pm 0.70$ & $35.3 \pm 1.0$ & $34.0 \pm 1.6$ & $33.2 \pm 1.3$ \\
$\beta$ & $0.184 \pm 0.066$ & $0.242 \pm 0.064$ & $0.106 \pm 0.044$ & $0.130 \pm 0.042$ \\
$\gamma$ & $\mathbf{2 . 1 4} \pm \mathbf{0 . 1 2}$ & $\mathbf{2 . 0 5 3} \pm \mathbf{0 . 0 9 4}$ & $\mathbf{2 . 2 9} \pm \mathbf{0 . 1 6}$ & $\mathbf{2 . 2 1} \pm \mathbf{0 . 1 1}$ \\
$s_{h}$ & $12.8 \pm 3.0$ & $16.41 \pm 0.64$ & 3.521 (fixed $)$ & 3.521 (fixed) \\
$K$ & - & $40 \pm 14$ & - & $39 \pm 12$ \\
\hline DOF & 159 & 234 & 160 & 235 \\
$\chi^{2} /$ DOF & 0.92 & 1.09 & 0.91 & 1.09 \\
$P\left(\chi^{2}\right)$ & 0.754 & 0.155 & 0.775 & 0.164 \\
\hline Figure: & - & 2 & - & 3 \\
\hline
\end{tabular}

\section{2. $\sqrt{s}_{\max }=8$ TeV ensemble}

For this ensemble, the unconstrained fits presented some disagreement with the selective criteria. In the case of global data reduction to $\sigma_{t o t}$ and $\rho$ the fit did not converge and therefore we have no solution for this case. In the individual fit to $\sigma_{\text {tot }}$ the data reduction converged but it should be noted that the corresponding error matrix is not positive definite.

The fit results and statistical information are displayed in Table 3 The curves, uncertainty regions and experimental information in the case of the constrained global fit to $\sigma_{\text {tot }}$ and $\rho$ data are shown in Fig. 4 (the unconstrained case did not converge).

\section{Discussion}

Using parametrizations (1)-(5), we are interested in a consistent quantitative description of the rise of the total cross-section at high energies. We have considered 
Table 3. $\sqrt{s}_{\max }=8 \mathrm{TeV}$ Ensemble. Fit results using Eqs. (1)-(3) for the $\sigma_{t o t}$ data (unconstrained, $s_{h}$ free, and constrained, $s_{h}=4 m_{p}^{2}$ ) and Eqs. (1)-(5) for the $\sigma_{\text {tot }}$ and $\rho$ data (only the constrained case). Units as in Table 1

\begin{tabular}{ccccc}
\hline & \multicolumn{2}{c}{ Unconstrained $(\delta$ free $)$} & \multicolumn{2}{c}{ Constrained $(\delta=1)$} \\
& $\sigma_{\text {tot }}$ & $\sigma_{\text {tot }}$ and $\rho$ & $\sigma_{\text {tot }}$ & $\sigma_{\text {tot }}$ and $\rho$ \\
\hline$a_{1}$ & $61.4 \pm 1.7$ & - & $60.8 \pm 6.9$ & $59.5 \pm 7.2$ \\
$b_{1}$ & $0.5062 \pm 0.0084$ & - & $0.530 \pm 0.061$ & $0.505 \pm 0.075$ \\
$a_{2}$ & $33.2 \pm 1.7$ & - & $33.2 \pm 2.3$ & $34.1 \pm 2.0$ \\
$b_{2}$ & $0.541 \pm 0.012$ & - & $0.541 \pm 0.016$ & $0.547 \pm 0.013$ \\
$\alpha$ & $32.37 \pm 0.22$ & - & $34.1 \pm 1.2$ & $33.4 \pm 1.8$ \\
$\beta$ & $0.0477 \pm 0.0022$ & - & $0.102 \pm 0.033$ & $0.124 \pm 0.054$ \\
$\gamma$ & $\mathbf{2 . 4 9 3} \pm \mathbf{0 . 0 1 6}$ & - & $\mathbf{2 . 3 0} \pm \mathbf{0 . 1 1}$ & $\mathbf{2 . 2 3} \pm \mathbf{0 . 1 5}$ \\
$s_{h}$ & $0.633 \pm 0.073$ & - & 3.521 (fixed) & 3.521 (fixed) \\
$K$ & - & - & - & $40 \pm 16$ \\
\hline DOF & 160 & - & 161 & 236 \\
$\chi^{2} /$ DOF & 0.91 & - & 0.91 & 1.09 \\
$P\left(\chi^{2}\right)$ & 0.774 & - & 0.788 & 0.172 \\
\hline Figure: & $5($ up $)$ & - & 5 (down) & 4 \\
\hline
\end{tabular}

8 variants of data reductions, obtaining full convergence in 7 cases. The fit results are displayed in Tables 2 and 3 . Our main goal is to investigate if these results indicate a log-squared behavior or a rise faster than this bound. In what follows, for a given numerical result $\gamma \pm \Delta \gamma$, we consider a result statistically consistent with a faster rise the cases in which $\gamma-\Delta \gamma>2$. In this case, for short, we will refer to a result statistically consistent with $\gamma>2$. However, as remarked early in Subs. 2.2.5. to consider the exponent $\gamma$ as a continuous real fit parameter has some special implications not present in the canonical assumption $\gamma=2$ (fixed). These aspects are directly related to the high-energy scaling factor $s_{h}$ and have important consequences not only on the data reductions but also on the physical interpretation of the fit results. The goal of this section is to address these aspects.

To this end, in Subsec. 4.1 we discuss the individual and global fit results, with focus on the value of the parameter $\gamma$ and its relation with a faster-than-squaredlogarithm rise. That will lead us to our partial conclusions in favor of this faster rise. After that, in Subsec. 4.2 we address the practical role and physical implications associated with the correlation between $\gamma$ and $s_{h}$. This discussion will lead us in Subsec. 4.3 to a final conclusion in favor of the constrained fits in both physical and statistical contexts, indicating a rise of the total cross-section faster than the log-squared bound.

\subsection{Individual and global fits: partial conclusions}

Based on the results displayed in Tables 2 and 3 we have the comments that follow.

In the case of individual fits to $\sigma_{t o t}$ data, all the results (constrained or unconstrained, $\sqrt{s}_{\max }=7$ or $8 \mathrm{TeV}$ ensembles) are statistically consistent with $\gamma>2$ (confirming, therefore, the conclusions first presented in Ref. 19). In all cases the integrated probability reads $P\left(\chi^{2}\right) \approx 0.8$. 
The highest $\gamma$-values are associated with the $\sqrt{s}_{\max }=8 \mathrm{TeV}$ ensemble, indicating $\gamma \approx 2.5$ (unconstrained fit) and $\gamma \approx 2.3$ (constrained fit). The corresponding results for $\sigma_{t o t}(s)$ and uncertainty regions are displayed in Fig. 5 for both the unconstrained and constrained fits, together with the experimental data. We notice that the agreement with the TOTEM measurements at $7 \mathrm{TeV}$ is striking. In the case of the constrained fit the uncertainty region includes the central value at $8 \mathrm{TeV}$ and also all the four $7 \mathrm{TeV}$ central values.

The numerical results and predictions for the total cross-section at some energies of interest are displayed in Table 4 (all the variants investigated in the individual fits). We also note that with the $\sqrt{s}_{\max }=8 \mathrm{TeV}$ ensemble, the predictions at 57 $\mathrm{TeV}$, namely $\sigma_{\text {tot }} \sim 142-143 \mathrm{mb}$ are about $7 \%$ larger than the central value of the result by the Pierre Auger Collaboration, namely $133 \mathrm{mb} \stackrel{35}{35}$

Table 4. Fit results and predictions for the $p p$ total cross-section at higher energies from individual fits to $\sigma_{\text {tot }}$ data.

\begin{tabular}{ccccc}
\hline & \multicolumn{2}{c}{$\sqrt{s}_{\max }=7 \mathrm{TeV}$} & \multicolumn{2}{c}{$\sqrt{s}_{\max }=8 \mathrm{TeV}$} \\
& Unconstrained & Constrained & Unconstrained & Constrained \\
\hline $7 \mathrm{TeV}$ & $97.8 \pm 1.1$ & $97.9 \pm 1.3$ & $98.12 \pm 0.86$ & $98.1 \pm 1.2$ \\
$8 \mathrm{TeV}$ & $100.2 \pm 1.2$ & $100.2 \pm 1.4$ & $100.56 \pm 0.91$ & $100.5 \pm 1.3$ \\
$14 \mathrm{TeV}$ & $110.6 \pm 1.6$ & $110.8 \pm 2.0$ & $111.4 \pm 1.1$ & $111.2 \pm 1.7$ \\
$57 \mathrm{TeV}$ & $140.6 \pm 3.2$ & $141.3 \pm 4.4$ & $142.7 \pm 1.8$ & $142.0 \pm 3.5$ \\
\hline
\end{tabular}

In the case of global fits to $\sigma_{t o t}$ and $\rho$ data, the results depend on the ensemble and on the constraint condition considered. In all cases of global convergent fits the integrated probability reads $P\left(\chi^{2}\right) \approx 0.2$. For the constrained fits (both ensembles) the results are statistically consistent with $\gamma>2$. In the unconstrained case and $\sqrt{s}_{\text {max }}=7 \mathrm{TeV}$ ensemble the result may be considered barely consistent with $\gamma>$ 2 , since, up to 2 figures, $\gamma$ lies in the interval 2.0 - 2.2. As stated before, for the $\sqrt{s}_{\text {max }}=8 \mathrm{TeV}$ ensemble we did not obtain full convergence.

The numerical results and predictions for the total cross-section at the energies of interest are displayed in Table 5. We note that at $57 \mathrm{TeV}$ the results indicate $\sigma_{\text {tot }} \sim 139-140 \mathrm{mb}$, which is about $5 \%$ larger than the Auger central value.

Table 5. Fit results and predictions for the $p p$ total cross-section at higher energies from global fits to $\sigma_{\text {tot }}$ and $\rho$ data.

\begin{tabular}{ccccc}
\hline & \multicolumn{2}{c}{$\sqrt{s}_{\max }=7 \mathrm{TeV}$} & \multicolumn{2}{c}{$\sqrt{s}_{\max }=8 \mathrm{TeV}$} \\
& Unconstrained & Constrained & Unconstrained & Constrained \\
\hline $7 \mathrm{TeV}$ & $97.5 \pm 1.2$ & $97.6 \pm 1.2$ & - & $97.8 \pm 1.3$ \\
$8 \mathrm{TeV}$ & $99.8 \pm 1.3$ & $99.9 \pm 1.3$ & - & $100.2 \pm 1.4$ \\
$14 \mathrm{TeV}$ & $109.9 \pm 1.7$ & $110.2 \pm 1.7$ & - & $110.6 \pm 2.0$ \\
$57 \mathrm{TeV}$ & $138.8 \pm 3.3$ & $139.6 \pm 3.4$ & - & $140.4 \pm 4.3$ \\
\hline
\end{tabular}

It is important to note that, in going from the individual to global fits, the constraint imposed by the inclusion of the $\rho$ information on the rise of $\sigma_{t o t}$ is evident: 
in all cases the $\gamma$ value decreases (Tables 2 and 3 ). In this respect, the subtraction constant plays a remarkable role due to its correlation with all the fit parameters in the nonlinear data reduction, specially those associated with the high-energy contribution, namely $\alpha, \beta, \gamma$ and $s_{h}$. This effect can be illustrated by the correlation matrix in the MINUIT Code, which provides a measure of the correlation between each pair of free parameters through a coefficient with numerical limits \pm 1 (full correlation) and 0 (no correlation) ${ }^{[28 \mid 32}$ For example, the coefficients in the global fits with the $\sqrt{s}_{\text {max }}=7 \mathrm{TeV}$ ensemble, in the cases of unconstrained fit (UF) and constrained fit $(\mathrm{CF})$, are displayed in Table 6 . In both cases the correlations between $K$ and $\alpha, \beta$ or $\gamma$ are around $0.8-0.9$, affecting therefore the asymptotic behavior of $\sigma_{\text {tot }}$. However, as already commented in Subsec.2.2.3 (and in more detail in Ref.20) this important parameter does not have a physical interpretation as is the case for those present in the parametrization of $\sigma_{t o t}(s)$. We also stress that the integrated probabilities $P\left(\chi^{2}\right)$ in the global fits are smaller than in the individual fits, $\sim 0.2$ and $\sim 0.8$, respectively.

Table 6. Correlation coefficients from the correlation matrices associated with unconstrained fit (UF) and constrained fit (CF) in the case of global fits with the $\sqrt{s}_{\max }=7 \mathrm{TeV}$ ensemble. The off-diagonal coefficients from the UF are displayed above the diagonal of the table (not filled) and those from the $\mathrm{CF}$, below that diagonal.

\begin{tabular}{c|c|ccccccccc}
\hline & & & & \multicolumn{1}{c}{ UF } & & & \\
\hline & & $a_{1}$ & $b_{1}$ & $a_{2}$ & $b_{2}$ & $\alpha$ & $\beta$ & $\gamma$ & $s_{h}$ & $K$ \\
\hline & $a_{1}$ & & 0.981 & 0.154 & 0.149 & 0.933 & -0.904 & 0.878 & -0.598 & 0.914 \\
& $b_{1}$ & 0.966 & & 0.092 & 0.094 & 0.985 & -0.969 & 0.950 & -0.539 & 0.909 \\
& $a_{2}$ & 0.193 & 0.129 & & 0.985 & 0.058 & -0.052 & 0.054 & 0.183 & -0.018 \\
$\mathrm{CF}$ & $b_{2}$ & 0.178 & 0.122 & 0.985 & & 0.063 & -0.059 & 0.062 & 0.187 & -0.038 \\
& $\alpha$ & 0.909 & 0.985 & 0.105 & 0.100 & & -0.997 & 0.988 & -0.443 & 0.877 \\
& $\beta$ & -0.847 & -0.949 & -0.125 & -0.122 & -0.986 & & -0.996 & 0.389 & -0.855 \\
& $\gamma$ & 0.825 & 0.933 & 0.126 & 0.123 & 0.976 & -0.998 & & -0.320 & 0.833 \\
& $s_{h}$ & - & - & - & - & - & - & - & & -0.543 \\
& $K$ & 0.882 & 0.872 & 0.000 & -0.032 & 0.835 & -0.787 & 0.770 & - & \\
\hline
\end{tabular}

Despite the aforementioned constraint, based on the discussion concerning the $\gamma$ values obtained in both individual and global fits, we conclude that our results favor a rise of the hadronic total cross-section faster than the log-squared behavior at the LHC energy region. By "favor" we mean that, within the uncertainties, the fit results lead to $\gamma$ values above 2 and not 2 or below 2. The results for the $\gamma$ parameter from all the fully converged fits, within the uncertainties (Tables 2 and 3), are schematically displayed in Figure 6. Note that, in the case of constrained fits, the $\gamma$ values lie in the interval $2.2-2.3$, which indicates more stability than in the unconstrained cases and suggests, therefore, a support to the former variant. We shall return to this point in section 4.3 . 


\subsection{The role and effects of the parameters $\gamma$ and $s_{h}$}

From the example displayed in Table 6 we also notice a substantial negative correlation between the parameter $\gamma$ and the energy scaling parameter $s_{h}$. In fact, from Tables 2 and 3, in going from the unconstrained to the constrained case (and also from individual to global fits), a decrease in $s_{h}$ is associated with an increase in $\gamma$ and vice versa.

In this section, based on the previous results and discussion, we examine in some detail the important practical and physical role of the scale parameter $s_{h}$, especially in what concerns data reductions with $\gamma$ as a free real (not integer) parameter. In what follows, we first list five characteristics of interest involved, distinguishing the case of $\gamma=2$, and then discuss the connections of these characteristics with our fit results, as well as with the COMPETE 2002 and PDG 2012 results (Table 1 and Figure 1).

1. Even treating $s_{h}$ as an unknown constant, with the canonical choice $\gamma=2$ the scaling factor can be well determined through the data reduction (since there is no correlation in this case). However, with $\gamma$ real and free we have two unknown and anticorrelated parameters. As a consequence, we can obtain statistically consistent solutions for different values of these parameters and that may imply in different physical pictures (as we shall show).

2. For $\gamma=2$ (fixed), the high-energy contribution $\sigma_{H E}(s)$, Eq. (3), is well defined for all values of $s$ as compared with the $s_{h}$ value. The only difference concerns the fact that for $s>s_{h}$ the logarithm term increases with the energy and for $s<s_{h}$ this contribution decreases as the energy increases (we will return to this important point in what follows). On the other hand, given that it represents a physical quantity, for $\gamma$ real (not integer) the logarithmic term is not defined for $s<s_{h}$ so that this component can only start at $s=s_{h}$. Above this point the contribution increases with the energy.

3. For $\gamma=2$ and $s_{h}=\delta\left[4 m_{p}^{2}\right]$ the high-energy contribution can be written

$$
\sigma_{H E}(s)=a+b \ln \left(\frac{s}{4 m_{p}^{2}}\right)+\beta \ln ^{2}\left(\frac{s}{4 m_{p}^{2}}\right),
$$

where $a=\alpha+\beta \ln ^{2}(\delta)$ and $b=-2 \beta \ln (\delta)$, providing the explicit correlation among the high-energy parameters. The above expansion, however, is not possible in the case of $\gamma$ real $(\neq 2)$ so that the correlations are somewhat hidden in the nonlinear data reductions.

4. As commented before, the $\sigma_{H E}(s)$ component accounts for the rise of the total cross-section at high energies and is associated with the Pomeron exchange. In the standard soft Pomeron concept this term is expected to increase with the energy, as is the case of the simple pole parametrization, $s^{\epsilon}$ with $\epsilon$ slightly greater than zero 36 39

5. Another aspect of interest concerns the minimum value of the energy above which a given parametrization is supposed to be applied, or valid. In this 
respect, as we will discuss, the energy cutoff for data reduction, $\sqrt{s}_{\text {min }}$, plays a central role, in connection with the corresponding energy scale, $\sqrt{s_{h}}$. Here, as in both COMPETE and PDG analyses, we have adopted $\sqrt{s}_{\text {min }}=5 \mathrm{GeV}$.

Based on the above characteristics, it is expected that, depending on the values of $s_{\min }$ and $s_{h}$, different physical interpretations can emerge in the cases of $\gamma=2$ (fixed) and $\gamma$ real (not integer). Let us discuss these aspects and their connection with our fit results and those from the COMPETE 2002 and PDG 2012 analyses.

\section{- $\gamma=2$ (fixed)}

In this case, if $s_{h}<s_{\min }$ then in the region of experimental data $\left(\sqrt{s} \geq \sqrt{s}_{\text {min }}\right)$, the high-energy component $\sigma_{H E}(s)$ increases with the energy, as expected in the standard concept of the soft Pomeron contribution. That is the case of the 2012 PDG version since, from Table 1 .

$$
s_{h}=16.21 \pm 0.16 \mathrm{GeV}^{2}<s_{\text {min }}=25 \mathrm{GeV}^{2} .
$$

On the other hand, if $s_{h}>s_{\text {min }}$ then in the interval $\sqrt{s}_{\text {min }} \leq \sqrt{s} \leq \sqrt{s_{h}}$, the $\sigma_{H E}(s)$ component decreases as the energy increases, suggesting a physical disagreement with the standard soft Pomeron contribution. That, however, is the case of the 2002 COMPETE result, since, from Table 1

$$
s_{h}=34.00 \pm 0.54 \mathrm{GeV}^{2}>s_{\text {min }}=25 \mathrm{GeV}^{2} .
$$

The dependence of $\sigma_{H E}(s)$ for the two cases above is shown in Fig. 7 in the energy interval $4 \mathrm{GeV} \leq \sqrt{s} \leq 7 \mathrm{GeV}$ that includes the energy cutoff $\sqrt{s}_{\min }=5$ $\mathrm{GeV}$.

In this respect, according to the COMPETE Collaboration! "One must note that in some processes, the falling $\ln ^{2}\left(s / s_{0}\right)$ term from the triple pole at $s<s_{0}$ is important in restoring the degeneracy of the lower trajectories at low energy. Hence the squared logarithm manifests itself not only at very high energies, but also at energies below its zero." However, even accepting this argument on a decreasing Pomeron contribution in the physical region considered, the fast rise of $\sigma_{H E}(s)$ as the energy decreases below $\sqrt{s_{h}}$ (Figure 17) and above the physical threshold $\left(2 m_{p}\right)$, remains, in our opinion, unexplained.

A practical or even pragmatic consequence of this COMPETE result is the agreement between their 2002 prediction (with $\gamma=2$ in accordance with the FroissartMartin bound) and the $7 \mathrm{TeV}$ TOTEM measurement. Or, in other words, this result is directly connected to the rather large value of $s_{h}$. By contrast, the PDG result with $\gamma=2$ and smaller $s_{h}$, which is consistent with a rise of $\sigma_{H E}(s)$ in the whole interval of energy investigated, lies below the TOTEM datum (compare Figures 1 and 7).

Summarizing, with $\gamma=2$ : (a) the COMPETE correctly describes the $7 \mathrm{TeV}$ 
TOTEM datum, but with a decreasing $\sigma_{H E}(s)$ contribution above the cutoff $s_{\min }$ up to $s_{h}$ and a increasing contribution as the energy decreases below $s_{\min }$ (strictly divergent as $s$ decreases); (b) in the PDG 2012 result, $\sigma_{H E}(s)$ increases in the whole energy-interval investigated (above the cutoff) but lies (within the uncertainty) below the TOTEM datum.

- $\gamma$ real (not integer)

For $\gamma$ not integer, as commented before, the $\sigma_{H E}(s)$ component is not defined at $\sqrt{s}<\sqrt{s_{h}}$. The contribution starts at $\sqrt{s}=\sqrt{s_{h}}$ with $\sigma_{H E}\left(s_{h}\right)=\alpha$ and from this point on it increases with the energy, as expected in the standard soft Pomeron concept. With respect to our fit results, the $\sigma_{H E}(s)$ component is well defined in the whole interval of energy investigated since $\sqrt{s}_{\text {min }}=5 \mathrm{GeV}$ and in all data reductions $s_{h}<s_{\min }$ (Tables 2 and 3 ).

\subsection{Conclusions on the best fit results}

Based on the physical aspects related to the scale factor, we now examine our unconstrained and constrained fit results in connection with our representation (6). This discussion, together with that in Subsec. 4.1, will lead us to conclude that the best results are those obtained with the constrained variant.

With the unconstrained fits ( $\delta$ or $s_{h}=\delta 4 m_{p}^{2}$ free) we have obtained for the $\sqrt{s}_{\text {max }}=7 \mathrm{TeV}$ ensemble $s_{h} \sim 13 \mathrm{GeV}^{2}$ (individual fit), $s_{h} \sim 16 \mathrm{GeV}^{2}$ (global fit) and for the $\sqrt{s}_{\text {max }}=8 \mathrm{TeV}$ ensemble $s_{h} \sim 0.6 \mathrm{GeV}^{2}$ (individual fit). In these cases it seems difficult to devise a physical meaning for the onset of the $\sigma_{H E}(s)$ component, because its value depends on the variant considered and data analyzed. Moreover, as mentioned in Subsec. 3.2. with the $\sqrt{s}_{\text {max }}=8 \mathrm{TeV}$ ensemble, the error matrix is not positive definite in the individual fit and no convergence was obtained in the global fit.

On the other hand, in the case of constrained fits $\left(s_{h}=4 m_{p}^{2}\right.$ fixed) the $\sigma_{H E}(s)$ component starts at this threshold with $\sigma_{H E}\left(s_{h}\right)=\alpha$ and from this point on it increases with the energy, namely $\sigma_{H E}(s) \geq \alpha$ at $\sqrt{s} \geq 2 m_{p}$. This threshold, $\sqrt{s_{h}} \sim 2 \mathrm{GeV}$, is also below the energy cutoff, $\sqrt{s}_{\text {min }}=5 \mathrm{GeV}$. This situation seems physically meaningful to us in both phenomenological and theoretical contexts. Furthermore, in all cases investigated with the constraint condition, especially with the $\sqrt{s}_{\max }=8 \mathrm{TeV}$ ensemble, we have obtained full convergence in the data reductions and consistent statistical results. As shown in Figure 6, the values of $\gamma$ are also consistent (stable) and lie around $2.2-2.3$, in all cases investigated with the constrained variant.

This discussion and the points raised in the previous sections favor, therefore, the results obtained with the constrained fits. Among them, we select as our best results the individual and global fits with the $\sqrt{s}_{\max }=8 \mathrm{TeV}$ ensemble. Both indicate a rise of the total cross-section that is faster than the log-squared behavior. 


\section{Fits to Elastic Cross-Section Data}

The total cross-section is related to the elastic cross-section in the forward direction via the optical theorem. That has led $\mathrm{us}^{20}$ to explore the possibility of extending the same analytical parametrization of the total cross-section, Eqs. (1)-(3), to the elastic (integrated) cross-section data, $\sigma_{e l}$. Based on unitarity, the same value of the exponent $\gamma$ obtained for $\sigma_{t o t}(s)$ is assumed for $\sigma_{e l}(s)$. For a detailed discussion on this assumption see section 3 in Ref. 20 .

\subsection{Fit and results}

The experimental data on $p p$ and $\bar{p} p$ scattering below $7 \mathrm{TeV}$ have been extracted from the PDG database $\frac{18}{18}$ without any kind of data selection or sieve procedure. The dataset includes also the recent TOTEM results (four points at $7 \mathrm{TeV}$ (Ref. 23) and one point at $8 \mathrm{TeV}$ (Ref. 24)). Statistical and systematic errors have been added in quadrature.

As feedback for initializing the parametrization of $\sigma_{e l}$ data we consider here the values of the parameters from our selected fit results to $\sigma_{\text {tot }}$ with both $\gamma$ and $s_{h}$ fixed (constrained fits in Table 3). We notice that the data reductions using as initial values the results from either the individual or global fits are similar. In what follows we focus mainly on the global case. The results with $\gamma=2.23$ and $s_{h}=$ $3.521 \mathrm{GeV}^{2}$ (fixed) are displayed in Table 7 and in Figure 8 (up) together with the evaluated uncertainty region.

\begin{tabular}{|c|c|}
\hline$a_{1}$ & $32.6 \pm 4.7$ \\
\hline$b_{1}$ & $0.579 \pm 0.044$ \\
\hline$a_{2}$ & $0.9 \pm 1.1$ \\
\hline$b_{2}$ & $0.41 \pm 0.31$ \\
\hline$\alpha$ & $4.74 \pm 0.15$ \\
\hline$\beta$ & $0.0380 \pm 0.0010$ \\
\hline$\gamma$ & 2.23 (fixed) \\
\hline$s_{h}$ & 3.521 (fixed) \\
\hline DOF & 102 \\
\hline$\chi^{2} / \mathrm{DOF}$ & 1.55 \\
\hline$P\left(\chi^{2}\right)$ & $3.33 \times 10^{-4}$ \\
\hline Figure: & 8 \\
\hline
\end{tabular}

From Table 7, the value of the $a_{2}$ parameter is statistically consistent with zero. This is a consequence of the equality of the $p p$ and $\bar{p} p$ elastic cross-sections data at low energies. We have checked that letting $a_{2}=0$, the same fit result is obtained. However, we notice that the statistical quality of the fit is not so good: 
large reduced $\chi^{2}$ and small integrated probability. Moreover, from Figure 8 , the fit uncertainty region barely reaches the extremum of the lower error bar of the TOTEM result at $8 \mathrm{TeV}$. On statistical grounds, since this point constitutes a high-precision measurement (defining the experimental information at the highest energy), the somewhat low fit quality may be associated with the underestimation of this datum by the fit result. We shall return to this important point related to the $8 \mathrm{TeV}$ TOTEM data in section 6.1.

Despite the limitations on the statistical quality of the fit, from Figure 8 (up), the global description of the experimental data seems satisfactory, including, within the uncertainties, the lower error bars of the four TOTEM results at $7 \mathrm{TeV}$. If we accept this data reduction as a reasonable description of the experimental data, the results, together with that obtained for the total cross-section, allow us to predict the ratio between the elastic and total cross-section as function of the energy. The result, within the uncertainties, is shown in Figure 8 (down), together with the experimental data. Using the $s$-channel unitarity, we have also included in this figure the result from the estimations of the total cross-section and the inelastic cross-section $\left(\sigma_{\text {inel }}\right)$ at $57 \mathrm{TeV}$ by the Auger Collaboration. ${ }^{35}$

\subsection{Asymptotic ratios}

The asymptotic ratio between the elastic and total cross-sections can be evaluated from parametrization (1)-(3). Denoting the parameters $\beta$ associated with the $\sigma_{t o t}$ and $\sigma_{e l}$ fits by the corresponding indexes, for $s \rightarrow \infty$, we have

$$
\frac{\sigma_{e l}}{\sigma_{t o t}} \rightarrow \frac{\beta_{e l}}{\beta_{t o t}} .
$$

From Tables 3 and 7 and the $s$-channel unitarity, we obtain

$$
\frac{\sigma_{e l}}{\sigma_{t o t}} \rightarrow 0.31 \pm 0.13 \quad \text { and } \quad \frac{\sigma_{\text {inel }}}{\sigma_{\text {tot }}} \rightarrow 0.69 \pm 0.13
$$

a result which is not in agreement with the naive black-disk model (limit 1/2), but statistically consistent, within the uncertainties, with rational limits

$$
\frac{\sigma_{e l}}{\sigma_{t o t}} \rightarrow \frac{1}{3} \quad \text { and } \quad \frac{\sigma_{\text {inel }}}{\sigma_{\text {tot }}} \rightarrow \frac{2}{3}
$$

as already obtained in our previous analysis, $\frac{20}{20}$ where only the first TOTEM measurement at $7 \mathrm{TeV}$ has been included. We note that from the individual fit to $\sigma_{\text {tot }}$ $\left(\gamma=2.30\right.$ and $s_{h}=3.521 \mathrm{GeV}^{2}$ fixed $)$ we obtain $\sigma_{e l} / \sigma_{t o t} \rightarrow 0.301 \pm 0.098$, a result also in agreement with (8), within the uncertainties.

These rational limits contrast with the prediction from the model-dependent amplitude analysis by Block and Halzen, which indicates the black-disk limit for both ratios ${ }^{40}$ However, the rational limits are not in disagreement with a saturation of the Pumplim bound, $\stackrel{41] 42}{4}$

$$
\frac{\sigma_{e l}}{\sigma_{t o t}}+\frac{\sigma_{d i f f}}{\sigma_{t o t}} \leq \frac{1}{2}
$$


where $\sigma_{\text {diff }}$ is the cross-section associated with the soft diffractive processes (single and double dissociation). This saturation and the rational limits corroborate the recent phenomenological arguments by Grau et al. who attribute the black-disk limit to the combination of the elastic and diffractive processes, calling also the attention to the possibility of the limiting value $1 / 3.43$ If that is the case, our results predict

$$
\frac{\sigma_{\text {diff }}}{\sigma_{\text {tot }}} \rightarrow \frac{1}{6} \quad \text { as } \quad s \rightarrow \infty
$$

\section{On a Fast Rise of the Total Cross-Section}

Our results with both ensembles $\left(\sqrt{s}_{\max }=7 \mathrm{TeV}\right.$ and $\left.8 \mathrm{TeV}\right)$ indicate the possibility of a rise of $\sigma_{\text {tot }}$ faster than the log-squared behavior. In addition, there seems to be some interesting aspects related to the data at the highest LHC energy that deserve further comments. In this section we first discuss some features of the 8 $\mathrm{TeV}$ TOTEM data $\left(\sigma_{t o t}\right.$ and $\left.\sigma_{e l}\right)$, as compared with those at $7 \mathrm{TeV}$ and in the region below this energy. After that, we present a few conjectures related to the possibility of a fast increase of the total cross-section at the LHC energy region and beyond.

\subsection{The 8 TeV TOTEM data}

From Tables 2 and 3, in going from the $\sqrt{s}_{\max }=7 \mathrm{TeV}$ ensemble to the $\sqrt{s}_{\max }$ $=8 \mathrm{TeV}$ ensemble, we can note a slight increase in the value of the $\gamma$ parameter (although consistent within the uncertainties in the constrained case). That may suggest a rise of $\sigma_{\text {tot }}$ in the $7-8 \mathrm{TeV}$ region faster than that observed up to $7 \mathrm{TeV}$. In this respect we draw the attention to the results that follows.

1. In all data reductions for $\sigma_{\text {tot }}$ presented here, the central values of the TOTEM data at $7 \mathrm{TeV}$ are well described within the uncertainties (Figures 2 - 5).

2. In the case of the $8 \mathrm{TeV}$ datum, the same results present good agreement only with the lower error bar and, in general, the fit uncertainty does not reach the central value. With our selected constrained results the uncertainty region includes the central value in the case of the individual fit (Figure 5) and only barely reaches this point in the case of the global fit (Figure 4).

3. Analogous effects can be observed in the case of the $\sigma_{e l}$ data: within the uncertainties the fit results are consistent with the lower error bars at $7 \mathrm{TeV}$, but reaches only the extremum of the lower bar at $8 \mathrm{TeV}$.

Therefore, even with $\gamma$ greater than 2 and the scaling parameter fixed or free, the fit results are not in statistical agreement with the $8 \mathrm{TeV}$ TOTEM data on $\sigma_{\text {tot }}$ and $\sigma_{e l}$ : the fits somewhat underestimate the high-precision experimental values, suggesting a rise faster than expected. In this respect, some quantitative inferences may be instructive, even if only in a limited context, as discussed in what follows. 
First, although associated with different variants, the results here obtained for the $\gamma$ parameter $\left(\gamma_{i} \pm \Delta \gamma_{i}\right)$ can be used to provide a quantitative information on typical values associated, separately, with the ensembles $\sqrt{s}_{\max }=7 \mathrm{TeV}$ (four points) and $\sqrt{s}_{\text {max }}=8 \mathrm{TeV}$ (three points). We have considered two evaluations, either a weighted mean (with weights $1 /\left[\Delta \gamma_{i}\right]^{2}$ ) or a fit by a constant function (MINUIT). The results are displayed in Table 8 showing that, from ensemble $\sqrt{s}_{\text {max }}$ $=7 \mathrm{TeV}$ to $\sqrt{s}_{\text {max }}=8 \mathrm{TeV}$, both evaluations indicate an increase in the value of $\gamma$ around $16 \%$.

Table 8. Global estimates for the average values of the parameter $\gamma$ from fits with ensembles $\sqrt{s}_{\max }=7 \mathrm{TeV}$ (Table 2, four points) and $\sqrt{s}_{\text {max }}=8 \mathrm{TeV}$ (Table 3 three points).

\begin{tabular}{ccc}
\hline Ensemble & Weighted mean & Constant function fit \\
\hline$\sqrt{s}_{\text {max }}=7 \mathrm{TeV}$ & $2.146 \pm 0.003$ & $2.15 \pm 0.06$ \\
$\sqrt{s}_{\text {max }}=8 \mathrm{TeV}$ & $2.4861 \pm 0.0003$ & $2.49 \pm 0.02$ \\
\hline
\end{tabular}

Second, in order to get some quantitative information directly related to the highest energy region, we have developed fits to $\sigma_{\text {tot }}$ data with only the high-energy parametrization $\sigma_{H E}$, Eq. (3), but applied to datasets with different energy cutoffs: $\sqrt{s}_{\text {min }}=62.5 \mathrm{GeV}$ (CERN-ISR), $546 \mathrm{GeV}$ (CERN-Collider) and $1.8 \mathrm{TeV}$ (Fermilab). As already selected, we have considered the constrained variant $\left(s_{h}=4 m_{p}^{2}\right.$ fixed) with $\sqrt{s}_{\text {max }}=8 \mathrm{TeV}$. In this case the parametrization has only three free fit parameters, namely $\alpha, \beta$ and $\gamma$. For the first cutoff $\left(\sqrt{s}_{\min }=62.5 \mathrm{GeV}\right)$ we have used as feedback the values of the parameters obtained in the individual fit to $\sigma_{\text {tot }}$ data in the constrained case and ensemble $\sqrt{s}_{\max }=8 \mathrm{TeV}$ (fourth column in Table $3)$. Then, the fit result has been used as feedback for the second cutoff $\left(\sqrt{s}_{\text {min }}=\right.$ $546 \mathrm{GeV}$ ) and the same process for the third one. The results with the first two cutoffs are displayed in Table 9 and Figure 9 (for $\sqrt{s}_{\text {min }}=1.8 \mathrm{TeV}$ the results are similar to those obtained with $\sqrt{s}_{\text {min }}=546 \mathrm{GeV}$ ).

From Figure 9 we note that in the case of $\sqrt{s}_{\min }=62.5 \mathrm{GeV}$ the fit result with only three parameters is in plenty agreement with all the $p p$ and $\bar{p} p$ experimental data above $\sim 30 \mathrm{GeV}$, describing also the $p p$ data at lower energies. The TOTEM data at 7 and $8 \mathrm{TeV}$ are also described within the uncertainties and in this case $\gamma \approx$ 2.5 (Table 9). With the cutoff $\sqrt{s}_{\text {min }}=546 \mathrm{GeV}$, the fit is in agreement with the high-energy data (above $\sim 100 \mathrm{GeV}$ ) and the TOTEM data is quite well described (especially at $8 \mathrm{TeV}$ ); however, in this case $\gamma \approx 3.3$ (Table 9).

We understand that all the aforementioned results and discussions seem to suggest a fast unexpected rise of the cross-sections from 7 to $8 \mathrm{TeV}$ as compared with the region below $7 \mathrm{TeV}$.

\subsection{Some conjectures}

At this point, we could conjecture (if not speculate) on the implication of a possible increase of $\sigma_{t o t}$ faster than $\ln ^{2} s$. One possibility points to a power-like behavior 


\begin{tabular}{|c|c|c|}
\hline$\sqrt{s}_{\text {min }}:$ & $62.5 \mathrm{GeV}$ & $546 \mathrm{GeV}$ \\
\hline$\alpha(\mathrm{mb})$ & $36.40 \pm 1.24$ & $45.68 \pm 1.34$ \\
\hline$\beta(\mathrm{mb})$ & $0.062 \pm 0.031$ & $0.0054 \pm 0.0014$ \\
\hline$\gamma$ & $2.46 \pm 0.17$ & $3.282 \pm 0.088$ \\
\hline DOF & 17 & 8 \\
\hline$\chi^{2} / \mathrm{DOF}$ & 1.70 & 1.04 \\
\hline$P\left(\chi^{2}\right)$ & 0.035 & 0.401 \\
\hline Figure: & 9 & 9 \\
\hline
\end{tabular}

$s^{\epsilon}, \epsilon>0$, which has always been an important and representative approach $\underline{44} \underline{47}$ Predictions from unitarized models, developed nearly 10 years ago and consistent with the first $7 \mathrm{TeV}$ TOTEM datum, are discussed, for example, in Refs. 48

A faster-than-squared-logarithm rise could also indicate the onset of some new physics effect at the LHC energy region. For example, one possible explanation for the short penetration depth, recently observed in ultra-high-energy cosmic rays (UHECRs) around $100 \mathrm{TeV}$, is just an increase of the proton cross-section faster than the extrapolations from models, which have been tested only at lower energies (see, for example, Refs. 53 and 54 and references therein). These conjectures are not in disagreement with the recent theoretical arguments by Azimov 5.7

At last, in contrast to an effective violation of the Froissart-Martin bound, a fast rise of the total cross-section may also be associated with some local effect at the LHC energy region and/or beyond, so that, asymptotically, the bound might remain valid. In that case, $\gamma$ could represent a kind of effective exponent, depending on the energy and, possibly, associated with sums of different high-energy contributions. However, if constituting only a local effect our asymptotic results for the ratios involving the cross-sections might not be valid.

\section{Conclusions and Final Remarks}

In 2002, Barone and Predazzi stated (Ref. 36, page 140):

"The issue of the exact growth with energy of the total cross-sections is both delicate and unresolved; the mild growth of total cross-sections could be simulated by essentially any form and logarithmic physics is exceedingly difficult to resolve in a clear cut way."

The aim of this paper has been to take one more step in our investigation on the rise of the total hadronic cross-section at high energies. As in our previous analyses $\frac{19 \mid 20}{20}$ we have employed the analytical parametrization introduced by Amaldi et $a l$., with the exponent $\gamma$ in the high-energy leading logarithm contribution treated as a free real parameter in nonlinear data reductions. 
Here, the main points consisted in an updated analysis (including in the dataset the recent high-precision TOTEM measurements at $7 \mathrm{TeV}$ and $8 \mathrm{TeV}$ ) and a discussion on the correlation, practical role and physical meaning associated to the exponent $\gamma$ and the energy scale factor $s_{h}$. As in our previous works, we have considered different variants, involving two ensembles, individual/global fits and unconstrained/constrained fits. As feedbacks for the nonlinear data reductions we have used the "conservative" values obtained by the PDG in the recent 2012 Review of Particle Physics edition.

In section 4 we have discussed all the fit results, indicating the advantages of the constrained fits ( $s_{h}=4 m_{p}^{2}$ fixed) in both phenomenological and theoretical contexts and noticing also the statistical consistence of the fit results. In particular, we have concluded that the constrained fits with the $\sqrt{s}_{\max }=8 \mathrm{TeV}$ ensemble (individual and global cases) represent our best results (Table 3, fourth and fifth columns and Figures 4 and 5). They indicate a rise of the total hadronic cross-section faster than the log-squared bound at the LHC energy region. The results and predictions for the $p p$ total cross-section at energies of interest are displayed in the last columns of Tables 4 and 5 . A critical discussion on the COMPETE 2002 prediction for the total cross-section and the recent 2012 PDG result has been also presented.

With the selected results mentioned above, extensions of the parametrization to fit the elastic cross-section data, with fixed $\gamma$, have led to almost satisfactory results. Asymptotic limits for the ratios between elastic/total and inelastic/total cross-sections indicate consistence with $1 / 3$ and $2 / 3$, respectively (in agreement with our previous result 20 ).

We have called the attention to a possible fast rise of the cross-section between $7 \mathrm{TeV}$ and $8 \mathrm{TeV}$, according to the TOTEM results. We have conjectured that a fast rise might be connected with the onset of some new phenomena and have also speculated on the possible connection with the short penetration depth recently observed in UHECRs. If these effects have a local character (finite energies), there might be no contradiction with the Froissart-Martin bound, since it has been derived for the asymptotic energy limit, $s \rightarrow \infty$.

At last, we mention our recently updated comparative analysis,, 55 which includes also fits with either $\gamma=2$ (fixed) or a simple pole parametrization for the high-energy contributions (namely $s^{\epsilon}, \epsilon>0$ ). Beyond further discussions on the effects associated with the parameters $\gamma$ and $s_{h}$ (not present in the simple pole parametrization), the results complement and corroborate those presented here and in the previous works. $19[20$

Our final conclusion, as we have stressed, is that the rise of the total hadronic cross-section at the highest energies still constitutes an open problem, demanding, therefore, further and detailed investigation. Updated amplitude analyses by other authors including in the dataset all the high precision TOTEM measurements at 7 $\mathrm{TeV}$ and $8 \mathrm{TeV}$ can provide further checks on the results we have obtained and the conclusions we have drawn. 


\section{Acknowledgments}

We are grateful to an anonymous referee for valuable comments and suggestions, especially in respect to section 6.1. We are thankfull to D.A. Fagundes and D.D. Chinellato for useful discussions. Research supported by FAPESP (Contracts Nos. 11/15016-4, 09/50180-0).

\section{References}

1. M. Froissart, Phys. Rev. 123, 1053 (1961).

2. A. Martin, Nuovo Cimento A 42, 930 (1966).

3. A. Martin, Nuovo Cimento A 44, 1219 (1966).

4. L. Lukaszuk and A. Martin, Nuovo Cimento A 52, 122 (1967).

5. Ya. I. Azimov, Phys. Rev. D 84, 056012 (2011).

6. Ya. I. Azimov, Froissart bounds for amplitudes and cross-sections at high energies, arXiv: 1204.0984 [hep-ph].

7. Ya. Azimov, What is the real meaning of the Froissart theorem?, arXiv: 1208.4304 [hep-ph].

8. COMPETE Collab. (J. R. Cudell et al.), Phys. Rev. D 65, 074024 (2002).

9. COMPETE Collab. (J. R. Cudell et al.), Phys. Rev. Lett. 89, N. 20, 201801 (2002).

10. K. Igi and M. Ishida, Phys. Rev. D 66, 034023 (2002).

11. K. Igi and M. Ishida, Phys. Lett. B 622, 286 (2005).

12. M. M. Block and F. Halzen, Phys. Rev. D 70, 091901 (2004).

13. M. M. Block and F. Halzen, Phys. Rev. D 72, 036006 (2005).

14. Particle Data Group (K. Nakamura et al.), J. Phys. G: Nucl. Part. Phys. 37, 075021 (2010).

15. TOTEM Collab. (G. Antchev et al.), Europhys. Lett. 95, 41001 (2011).

16. TOTEM Collab. (G. Antchev et al.), Europhys. Lett. 96, 21002 (2011).

17. TOTEM Collab. (G. Antchev et al.), Europhys. Lett. 98, 31002 (2012).

18. Particle Data Group (J. Beringer et al.), Phys. Rev. D 86, 010001 (2012), http://pdg.lbl.gov

19. D. A. Fagundes, M. J. Menon and P. V. R. G. Silva, Braz. J. Phys. 42, 452 (2012), arXiv:1112.4704 [hep-ph].

20. D. A. Fagundes, M. J. Menon and P. V. R. G. Silva, J. Phys. G: Nucl. Part. Phys. 40, 065005 (2013), arXiv:1208.3456 [hep-ph].

21. U. Amaldi et al., Phys. Lett. B 66, 390 (1977).

22. TOTEM Collab. (G. Antchev et al.), Europhys. Lett. 101, 21002 (2013).

23. TOTEM Collab. (G. Antchev et al.), Europhys. Lett. 101, 21004 (2013).

24. TOTEM Collab. (G. Antchev et al.), Phys. Rev. Lett. 111, 012001 (2013).

25. UA4/2 Collab. (C. Augier et al.), Phys. Lett. B 315, 503 (1993).

26. K. Kang and B. Nicolescu, Phys. Rev. D 11, 2461 (1975).

27. R. F. Ávila and M. J. Menon, Nucl. Phys. A 744, 249 (2004).

28. P. R. Bevington and D. K. Robinson, Data Reduction and Error Analysis for the Physical Sciences, 2nd edn. (McGraw-Hill, Boston, Massachusetts, 1992).

29. D. A. Fagundes, M. J. Menon and P. V. R. G. Silva, Reply to "Commentary on Total Hadronic Cross-Section Data and the Froissart-Martin Bound by Fagundes, Menon and Silva", arXiv:1211.3352 [hep-ph].

30. R. F. Ávila, E. G. S. Luna and M. J. Menon, Braz. J. Phys. 31, 567 (2001).

31. R. F. Ávila, E. G. S. Luna and M. J. Menon, Phys. Rev. D 67, 054020 (2003).

32. ROOT Framework, http://root.cern.ch/drupal/; 

http://root.cern.ch/root/html/TMinuit.html

33. F. James, MINUIT Function Minimization and Error Analysis, Reference Manual, Version 94.1, CERN Program Library Long Writeup D506 (CERN, Geneva, Switzerland, 1998).

34. A. K. Common, Nuovo Cimento 69, 115 (1970).

35. The Pierre Auger Collaboration (P. Abreu et al.), Phys. Rev. Lett. 109, 062002 (2012).

36. V. Barone and E. Predazzi, High-Energy Particle Diffraction (Spring-Verlag, Berlin, 2002).

37. S. Donnachie, G. Dosch, P. V. Landshoff and O. Natchmann, Pomeron Physics and QCD (Cambridge University Press, Cambridge, 2002).

38. A. Donnachie and P. V. Landshoff, Z. Phys. C 2, 55 (1979).

39. A. Donnachie and P. V. Landshoff, Nucl. Phys. B 244, 322 (1984).

40. M. M. Block and F. Halzen, Phys. Rev. D 86, 051504 (2012).

41. J. Pumplin, Phys. Rev. D 8, 2899 (1973).

42. U. P. Sukhatme and F. S. Henyey, Nucl. Phys. B 108, 317 (1976).

43. A. Grau, S. Pacetti, G. Pancheri and Y. S. Srivastava, Phys. Lett. B 714, 70 (2012).

44. A. Donnachie and P. V. Landshoff, Phys. Lett. B 296, 227 (1992).

45. P. V. Landshoff, Acta Phys. Polon. B 40, 1967 (2009).

46. P. V. Landshoff, AIP Conf. Proc. 1105, 236 (2009), arXiv:0811.0260 [hep-ph]

47. A. Donnachie and P. V. Landshoff, Elastic Scattering at the LHC, arXiv:1112.2485 [hep-ph]

48. B. Z. Kopeliovich, I. K. Potashnikova, B. Povh and E. Predazzi, Phys. Rev. Lett. 85, $507(2000)$.

49. B. Z. Kopeliovich, I. K. Potashnikova and B. Povh, Phys. Rev. D 86, 051502(R) (2012).

50. V. A. Petrov and A. V. Prokudin, Eur. Phys. J. C 23, 135 (2002);

51. V. A. Petrov, E. Predazzi and A. Prokudin, Eur. Phys. J. C 28, 525 (2003).

52. V. A. Petrov and A. Prokudin, Three Pomerons vs DO and TOTEM Data, arXiv:1212.1924 [hep-ph].

53. N. Shaham and T. Piran, Phys. Rev. Lett. 110, 021101 (2013), arXiv:1204.1488 [astroph.HE].

54. R. Conceição, J. Dias de Deus and M. Pimenta, Nucl. Phys. A 888, 58 (2012), arXiv:1107.0912 [hep-ph].

55. M. J. Menon and P. V. R. G. Silva, A Study on Analytic Parametrizations for the Proton-Proton Cross-Sections and Asymptotia, arXiv:1305.2947 [hep-ph]. 


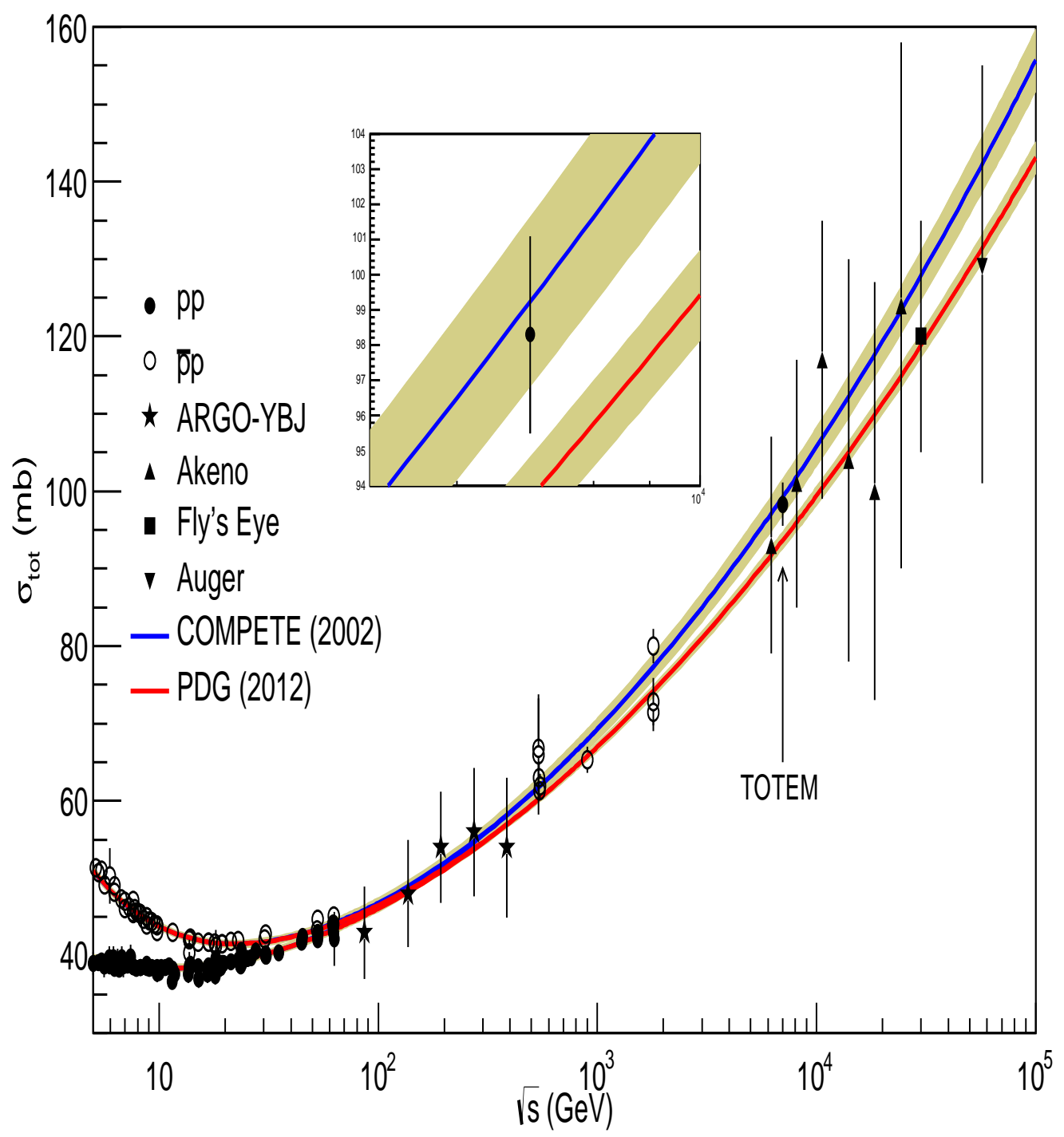

Fig. 1. Results with the COMPETE highest-rank parametrization (Eqs. (1)-(3) with $\gamma=2$ ) from the 2002 COMPETE analysis, $\frac{[8]}{9}$ and from the 2012 PDG version $[18$ (which includes the first 7 TeV TOTEM datum). The corresponding values of the parameters are displayed in Table 1 

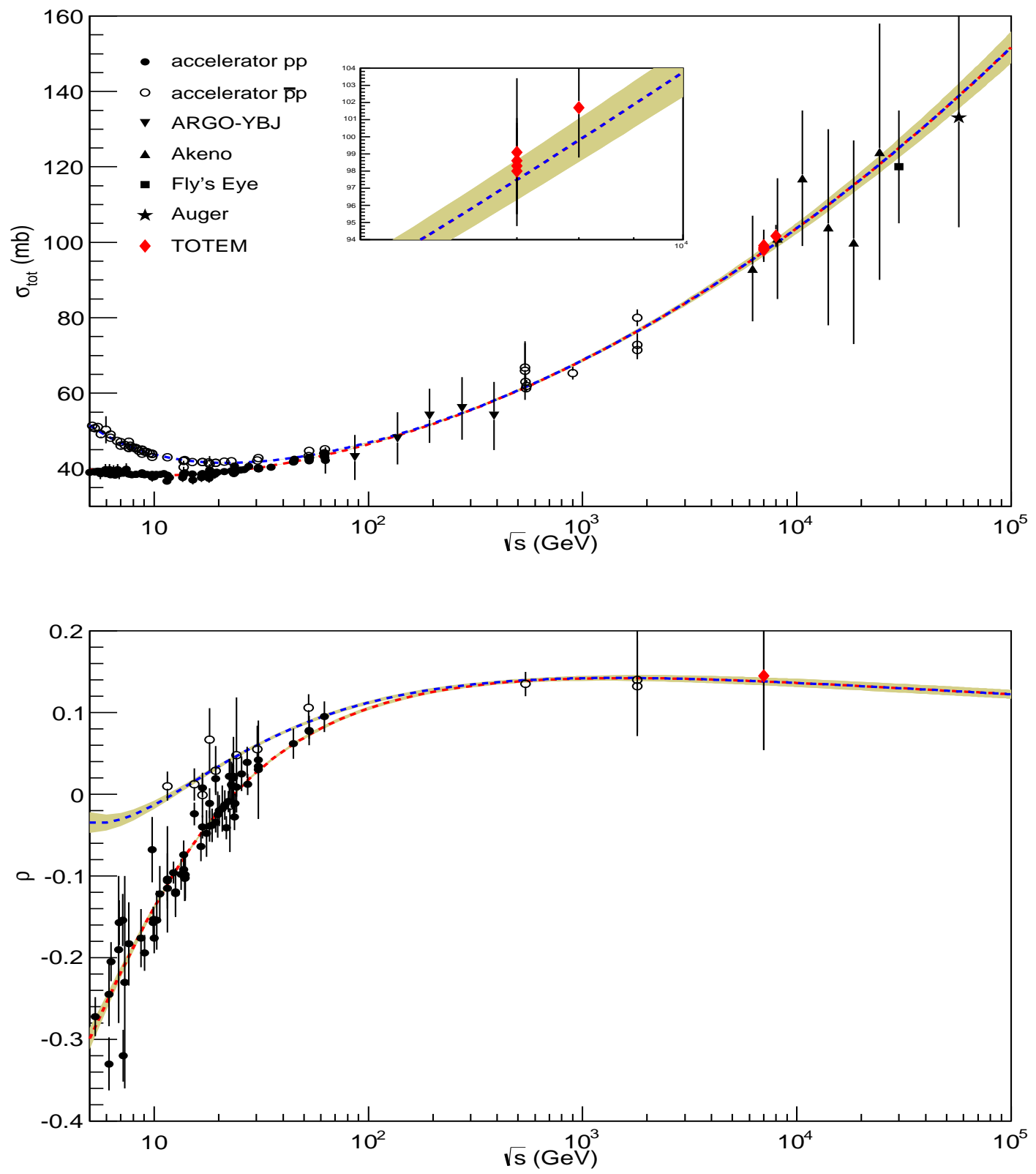

Fig. 2. Global unconstrained fit results with the $\sqrt{s}_{\text {max }}=7 \mathrm{TeV}$ ensemble (third column in Table 21. 

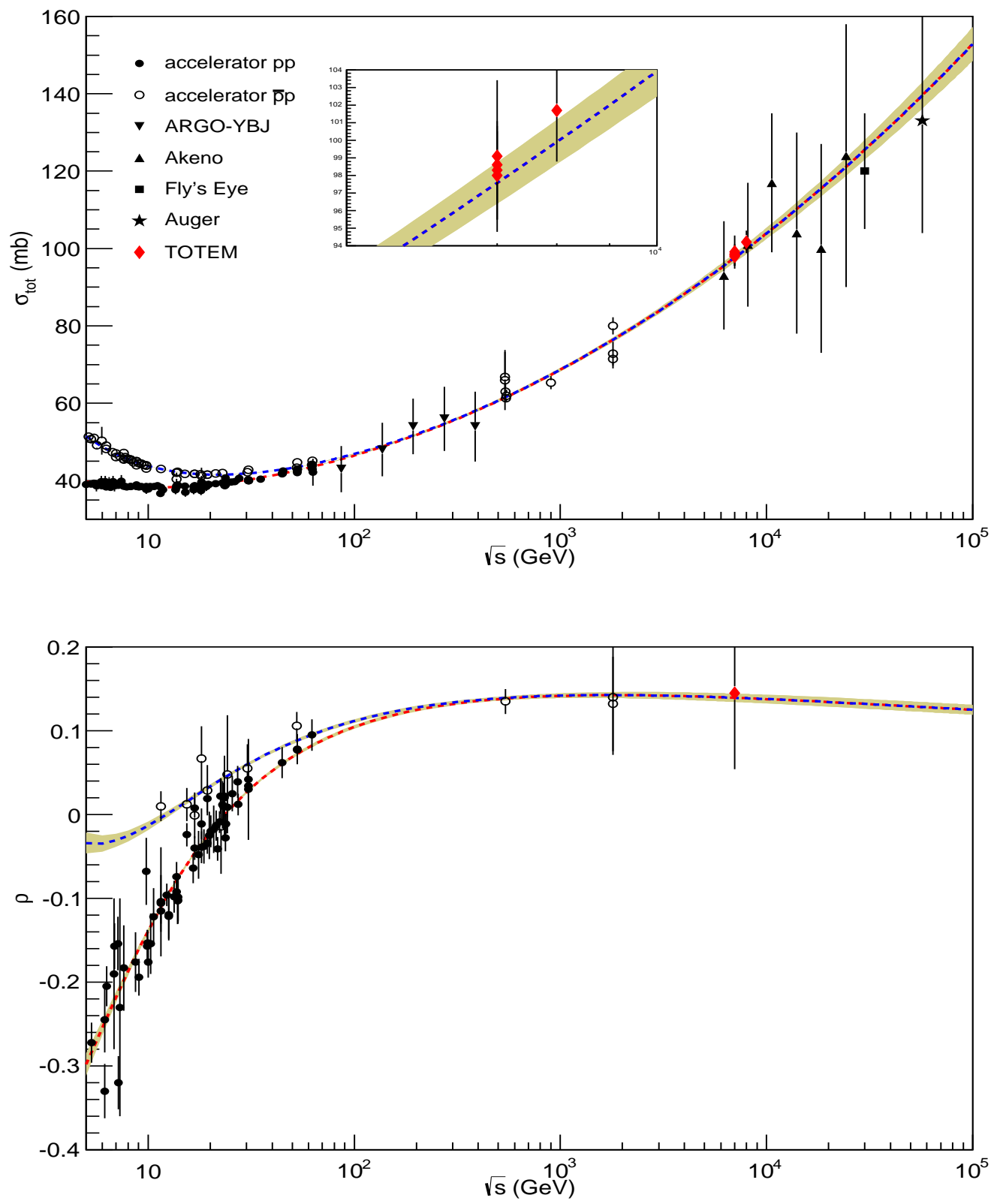

Fig. 3. Global constrained fit results with the $\sqrt{s}_{\text {max }}=7 \mathrm{TeV}$ ensemble (fifth column in Table 2). 

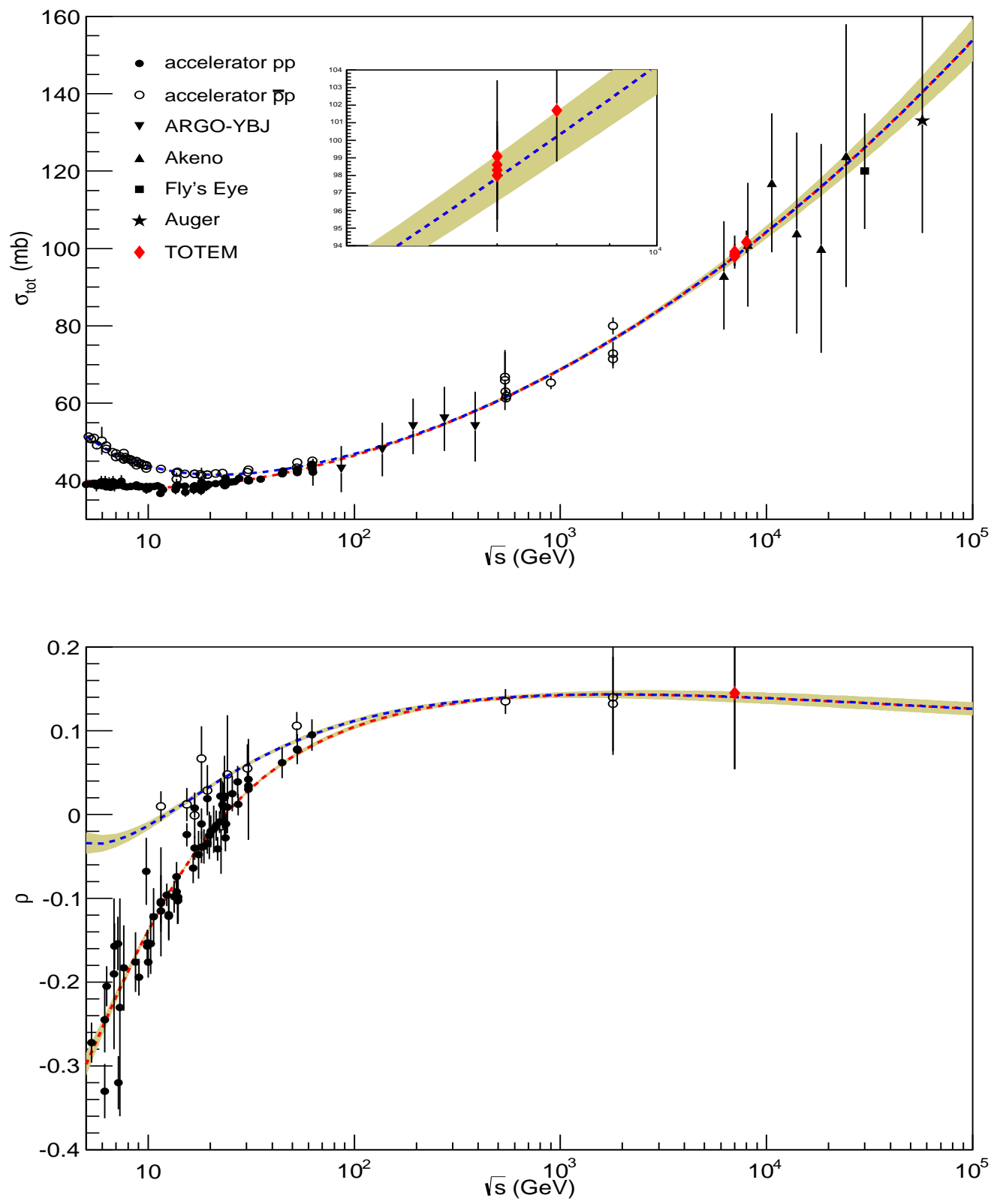

Fig. 4. Global constrained fit results with the $\sqrt{s}_{\text {max }}=8 \mathrm{TeV}$ ensemble (fifth column in Table 3). 

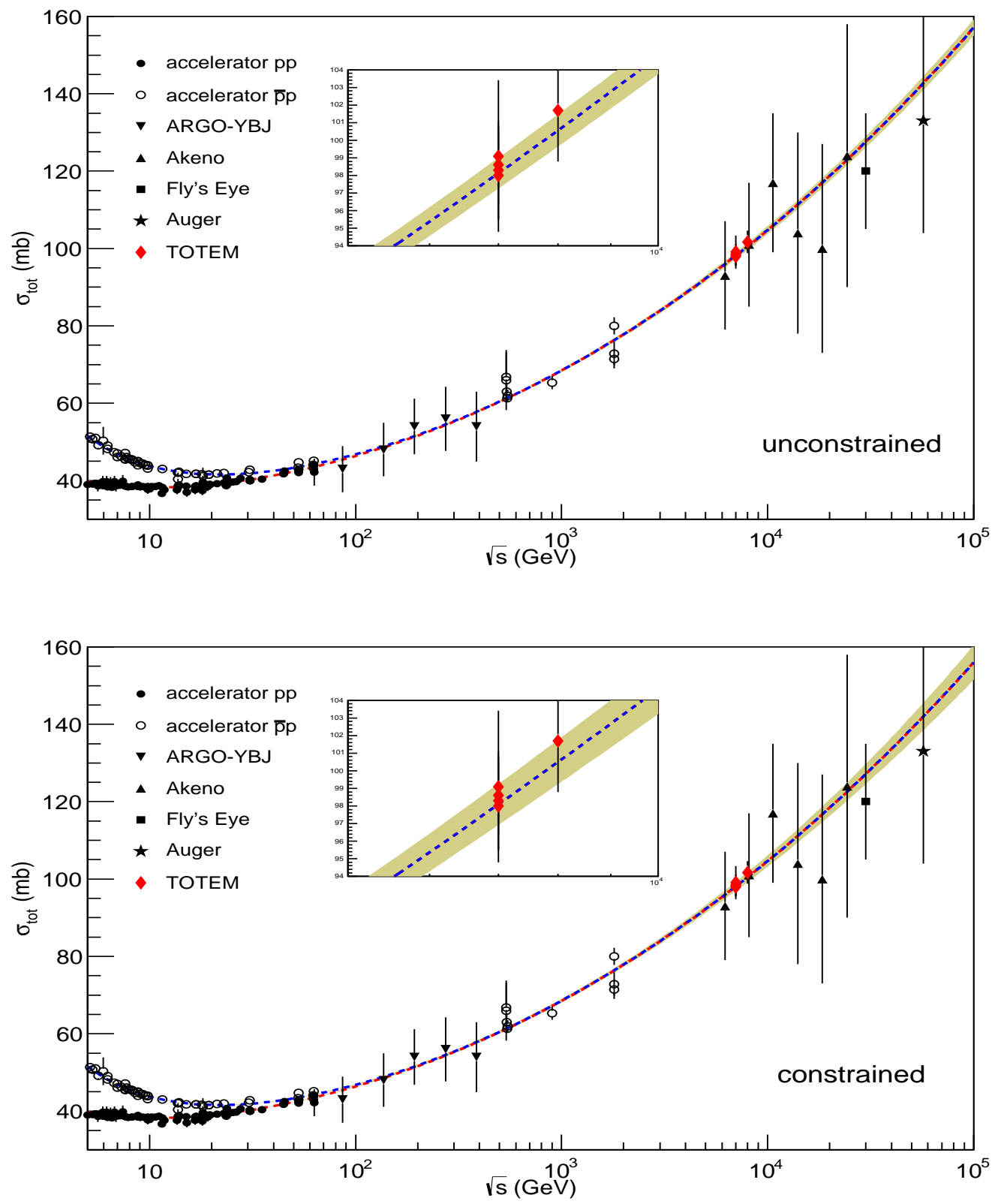

Fig. 5. Individual fit results to $\sigma_{\text {tot }}$ data with the $\sqrt{s}_{\text {max }}=8 \mathrm{TeV}$ ensemble and unconstrained (up) and constrained (down) data reductions (second column and fourth column in Table 3 respectively). 


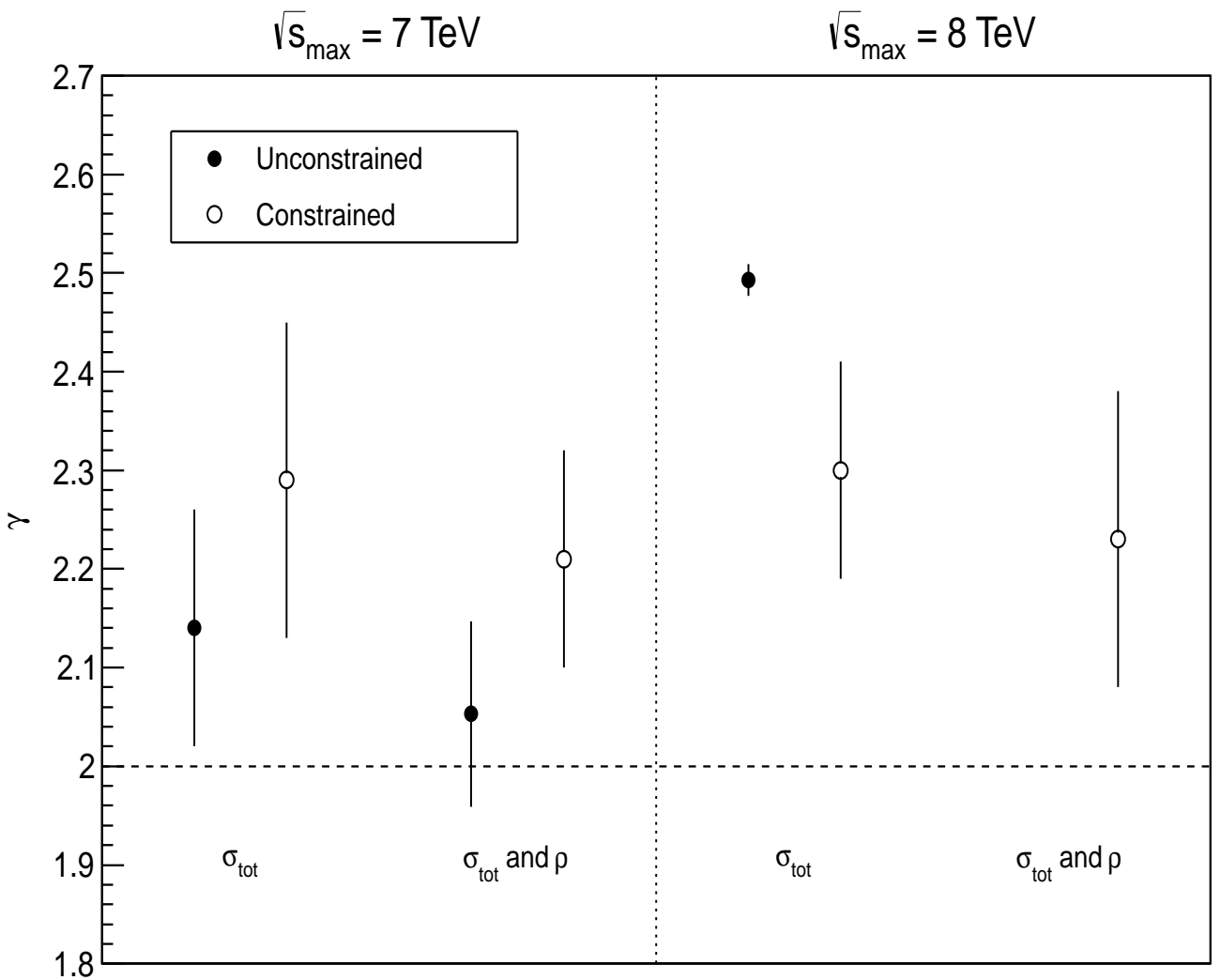

Fig. 6. Results obtained here for the exponent $\gamma$ as a free parameter in different data reductions through parametrization (1)-(3) and (1)-(5): ensembles $\sqrt{s}_{\max }=7 \mathrm{TeV}$ (Table 2) and $\sqrt{s}_{\max }=$ $8 \mathrm{TeV}$ (Table 3), constrained and unconstrained fits, individual $\left(\sigma_{t o t}\right)$ and global $\left(\sigma_{\text {tot }}\right.$ and $\rho$ ) fits. 

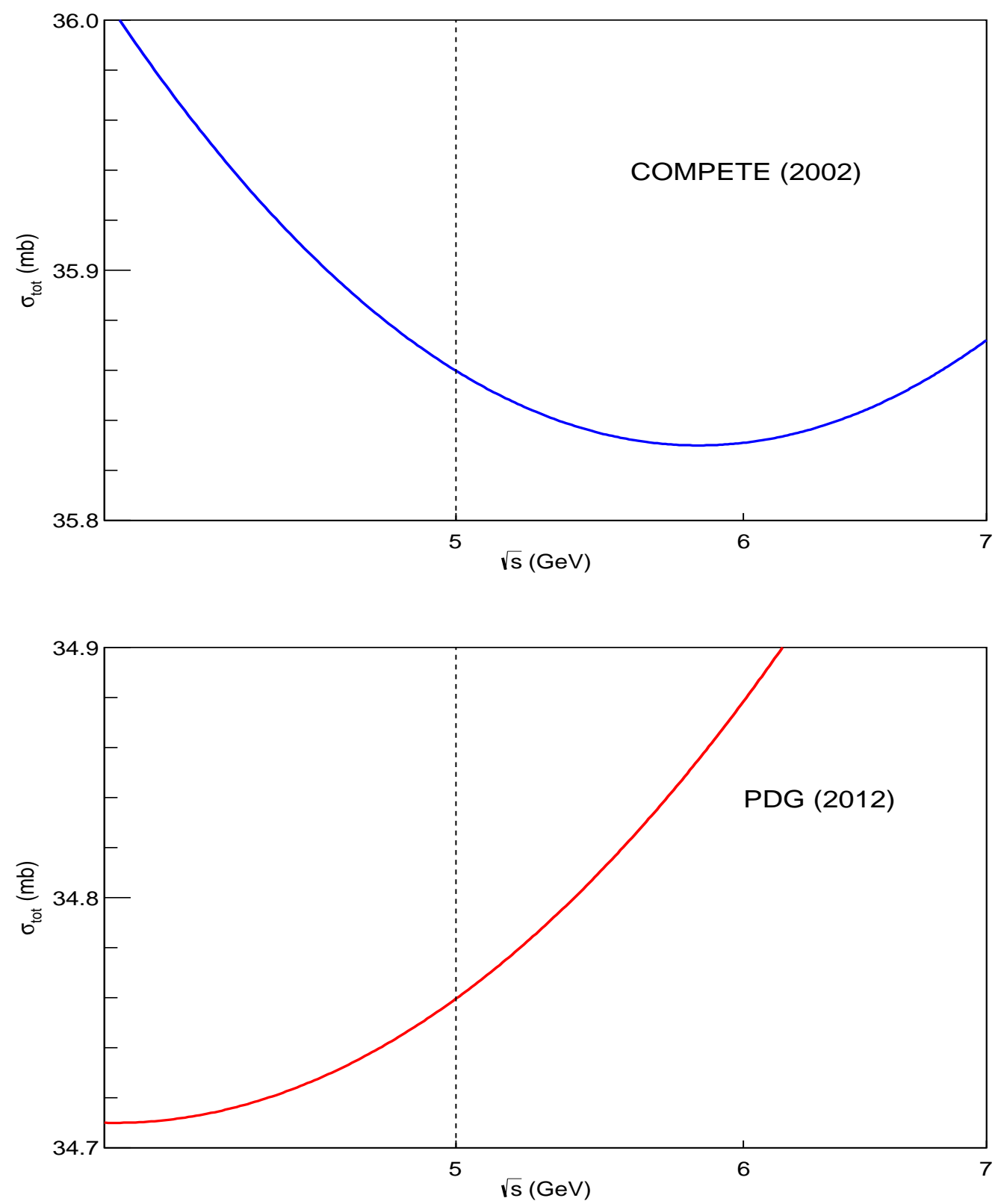

Fig. 7. High energy contribution $\sigma_{H E}(s)=\alpha+\beta \ln ^{2}\left(s / s_{h}\right)$ from the COMPETE 2002 and PDG 2012 analyses, around the energy cutoff $\sqrt{s}_{\text {min }}=5 \mathrm{GeV}$ (parameters from Table 1). 

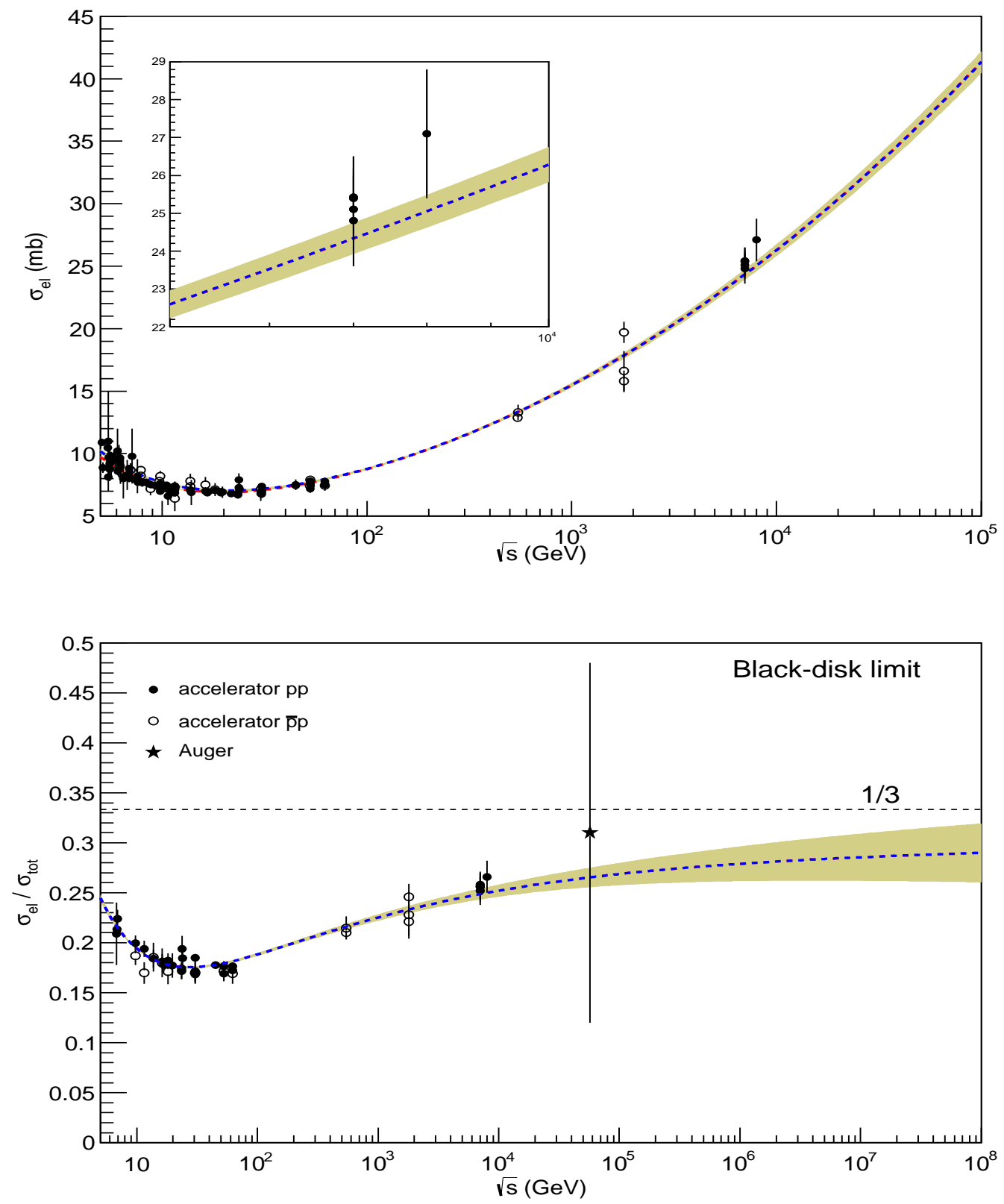

Fig. 8. Fit result for the elastic cross-section data (up) and predictions for the ratio between the elastic and total cross-sections (down). 

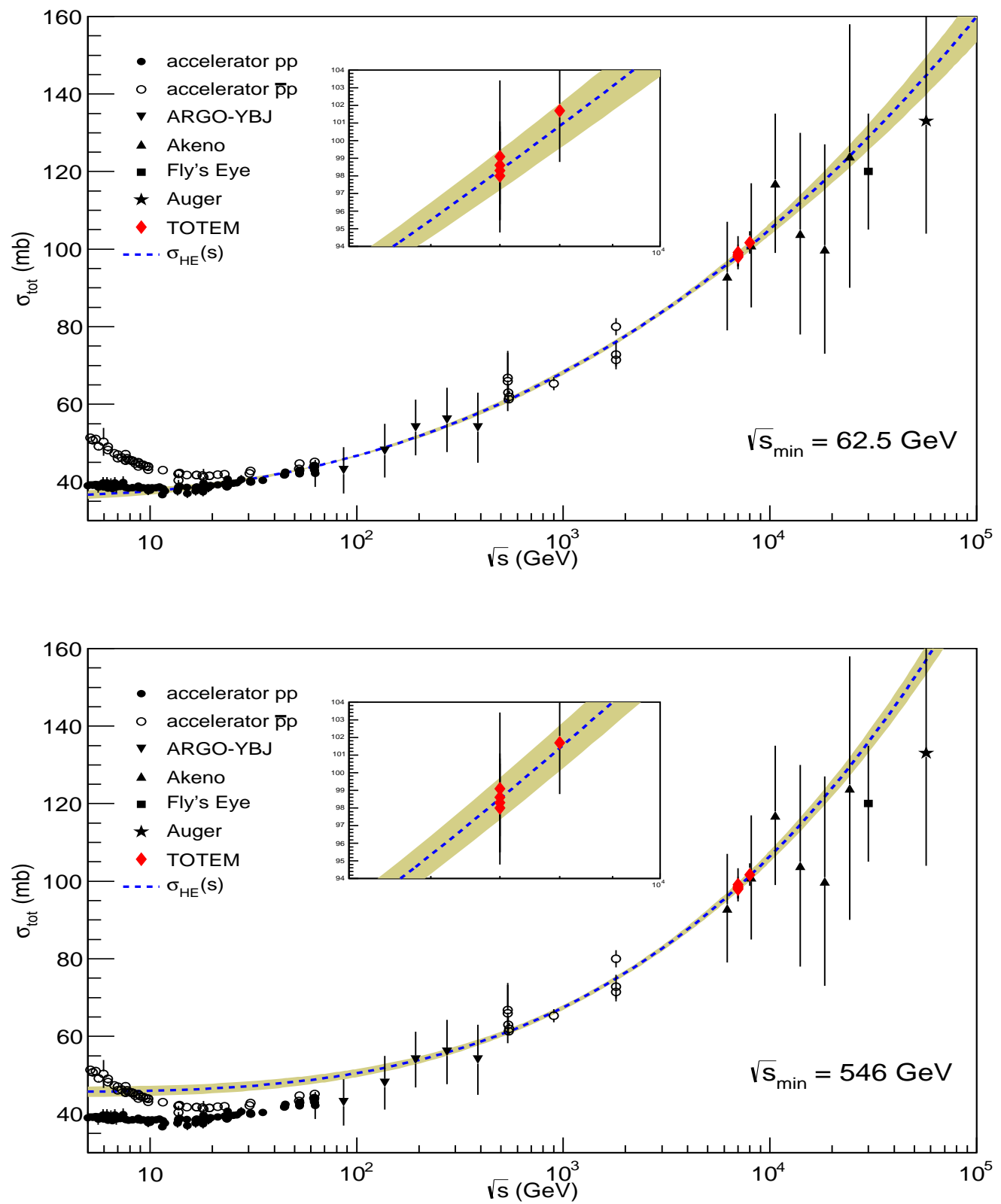

Fig. 9. Results of the constrained fit ( $s_{h}$ fixed) to ensemble $\sqrt{s}_{\max }=8 \mathrm{TeV}$ through parametrization $\sigma_{\mathrm{HE}}(s)$, Eq. (3), and two energy cutoffs, $\sqrt{s}_{\text {min }}=62.5 \mathrm{GeV}$ and $\sqrt{s}_{\text {min }}=546 \mathrm{GeV}$ (Table $9)$. 